\title{
World Computer \\ Chess Championship
}

RESULTS AND GAMES (KEN THOMPSON)

Köln, West Germany

June $11-15,1986$

\begin{tabular}{|c|c|c|c|c|c|c|c|c|}
\hline & rate & perf & 1 & 2 & 3 & 4 & 5 & total \\
\hline 1 Cray Blitz & 2200 & 2290 & $20+\square$ & $6--$ & $3+\square$ & $14+$ & $2+\square 4$ & \\
\hline 2 Hitech & 2270 & 2303 & $18+$ - & $14+\square$ & $7+$ - & $5+\square$ & $1-\square$ & \\
\hline 3 Bebe & 2100 & 2215 & $16+$ & $15+\square$ & $1-$ - & $11+\square$ & $5+\square$ & \\
\hline 4 Sun Phoenix & 0 & 2318 & $5-\square$ & $11+\square$ & $18+\square$ & $7+\square$ & $6+\square$ & \\
\hline 5 Rebel & 0 & 2235 & $4+\square$ & $12+$ - & $6+\square$ & $2-m$ & $3-\square 3$ & \\
\hline 6 Bobby & 0 & 2188 & $19+\square$ & $1+\square$ & $5-$ - & $8+\square$ & $4-\square 3$ & \\
\hline 7 Plymate & 0 & 2102 & $21+\square$ & $8+\square$ & $2-\square$ & $4-\mathbf{m}$ & $12+\square 3$ & 3 \\
\hline 8 Mephisto & 0 & 1973 & $9+\square$ & $7-$ & $17+\square$ & $6-$ & $14+\square 3$ & 3 \\
\hline 9 Dutch & 0 & 1828 & $8-=$ & $19+\square$ & $11=\mathbf{m}$ & $15=\square$ & $13+\square 3$ & 3 \\
\hline 10 Nona & 0 & 1552 & $14-=$ & $18-\square$ & $21+$ - & $22+$ & $15+\square 3$ & 3 \\
\hline 11 Advance $68 \mathrm{~K}$ & 0 & 1855 & $17+\square$ & $4-\square$ & $9=\square$ & $3-$ & $19+\square ?$ & $2^{1 / 2}$ \\
\hline 12 Lachex & 0 & 1840 & $13+$ & $5-\square$ & $16=$ & $18+\square$ & $7-\square$ & $2 \frac{1 / 2}{2}$ \\
\hline 13 Ostrich & 1750 & 1689 & $12-\square$ & $20+$ & $15=$ & $16+\square$ & $9-\mathbf{a}$ & $2^{1 / 2}$ \\
\hline 14 Schach 2.7 & 0 & 1716 & $10+\square$ & $2-$ & $22+\square$ & $1-\square$ & $8-m$ & \\
\hline 15 Cyrus 68K & 0 & 1572 & $22+\square$ & $3-=$ & $13=\square$ & $9=$ & $10-$ - & 2 \\
\hline 16 Vaxchess & 0 & 1561 & $3-\square$ & $23+$ & $12=\square$ & $13-$. & $17=\square$ & 2 \\
\hline 17 Chat & 0 & 1533 & $11-\mathbf{a}$ & $21+\square$ & $8-\pi$ & $19=\square$ & $16=$ & 2 \\
\hline 18 ВСР & 0 & 1645 & $2-\square$ & $10+\mathbf{m}$ & $4-\square$ & $12-$. & $20=\square$ & $1 \frac{1 / 2}{2}$ \\
\hline 19 Enterprise & 0 & 1591 & $6-\square$ & 9-. & $20+\square$ & $17=$ & $11-\square$ & $1 \frac{1 / 2}{2}$ \\
\hline 20 Awit & 1600 & 1476 & $1-$ & $13-\square$ & $19-$ & $21+\square$ & $18=$ " & $1 \frac{1 / 2}{2}$ \\
\hline $21 \operatorname{Rex}$ & 0 & 1157 & $7-\square$ & $17-\mathbf{m}$ & $10-\square$ & $20-$ - & $22+\square$ & 1 \\
\hline 22 Shess & 1000 & 855 & $15-$ & + & $14-$ & $10-\square$ & $21-\square$ & \\
\hline 23 Kempelen & 0 & 767 & - & $16-\square$ & & & & 0 \\
\hline
\end{tabular}

\section{Round 1}

Rex - Plymate

C22/01 04 Qa4

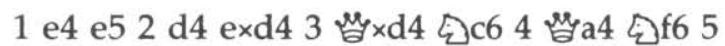
\&g5 \&c5 6 \&e2 h6 7 \&f4 O-O 8 §c3 月e8 9

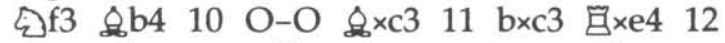

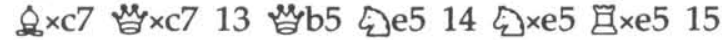

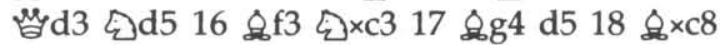

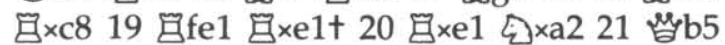

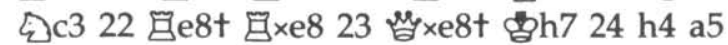

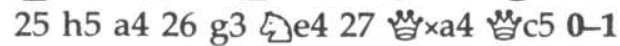

$$
\begin{gathered}
\text { Vaxchess - Bebe } \\
\text { B73/03 09 N:c6 }
\end{gathered}
$$

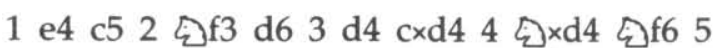
¿c3 g6 6 \&e2 \&g7 7 \&e3 $气$ c6 8 O-O O-O

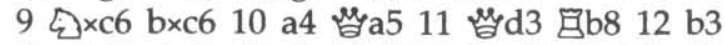

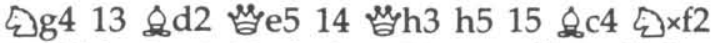

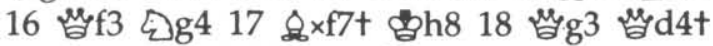

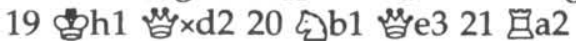

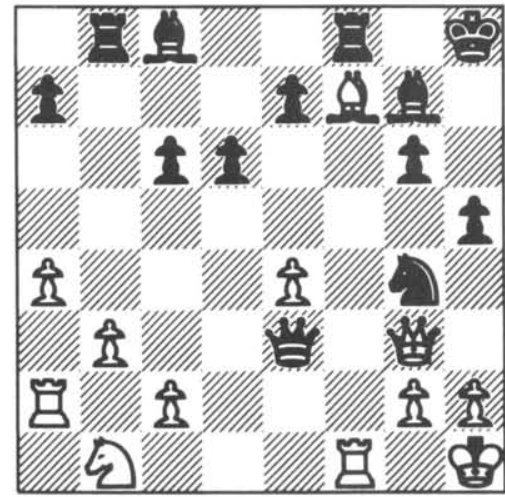

Position after 21 目a2

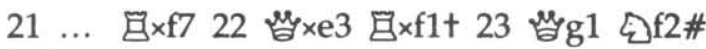
0-1

$$
\begin{gathered}
\text { Ostrich - Lachex } \\
\text { C68/05 07. Nf6 }
\end{gathered}
$$

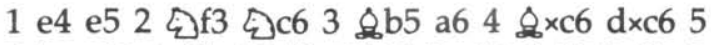

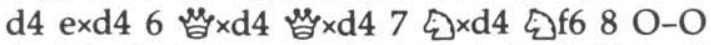
\&c5 9 c3 O-O 10 f3 \&d6 11 \&g5 c5 12 \& x6 


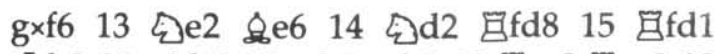

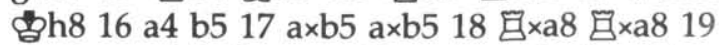

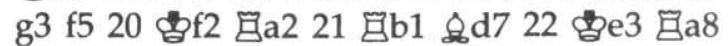

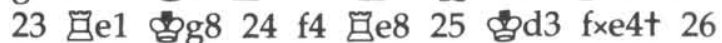
¿xe4 \&ff $27 \quad$ ¿c1 \&f8 28 b3 c4t 29 bxc4

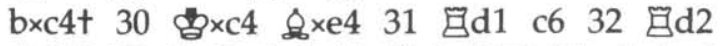

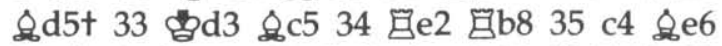

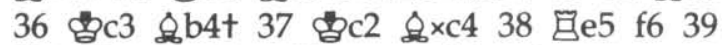

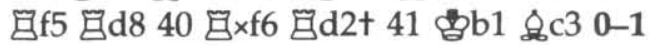

\section{Cray Blitz - Awit C42/09 10. Be6}

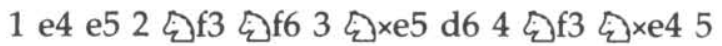

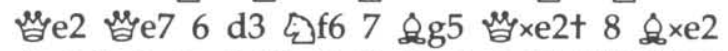
\&e7 9 ¿िc3 c6 10 O-O-O \&e6 11 Gde1 h6

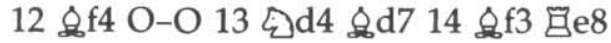

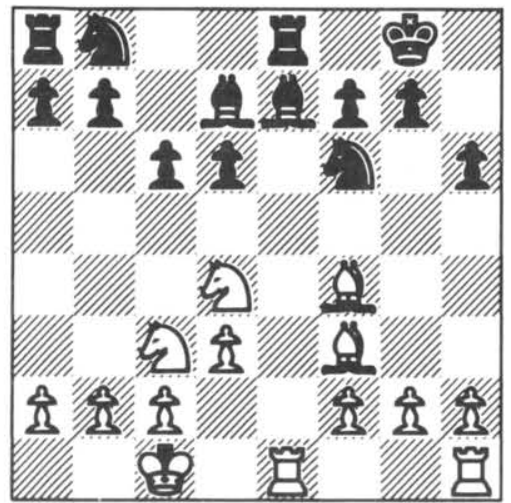

Position after $14 \ldots$...

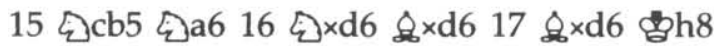

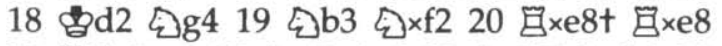

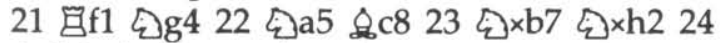

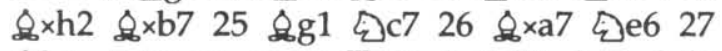
\&h5 c5 28 g3 g6 29 Ixf7 \&e4 30 \&g4 \&d5

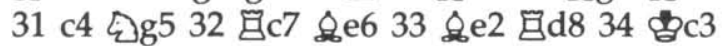

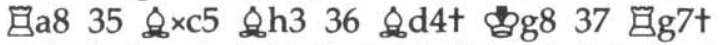

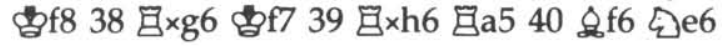
1-0

\section{Advance 68K - Chat C96/16 15. Qb6}

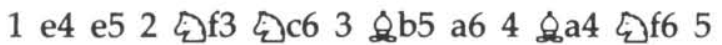
$\mathrm{O}-\mathrm{O}$ \&e7 6 目e1 b5 7 \&3 O-O 8 c3 d6 9

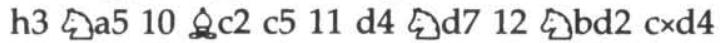

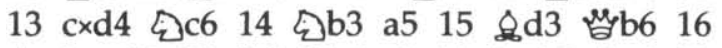

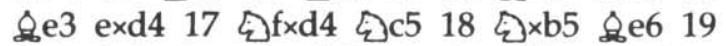

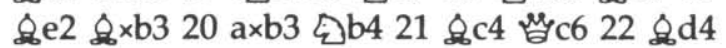

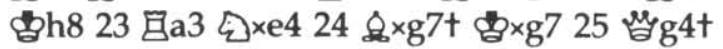

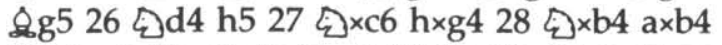

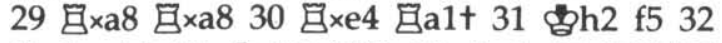

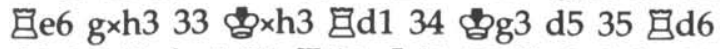

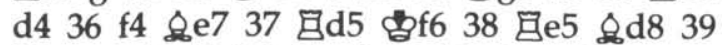

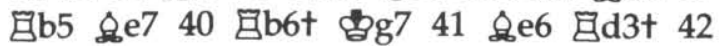

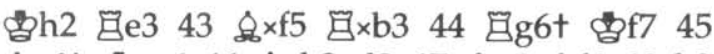

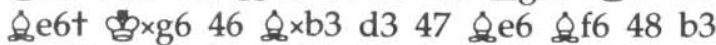

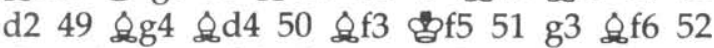
tgig1 \&7 53 की

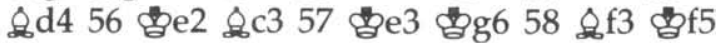

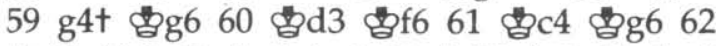

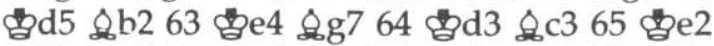

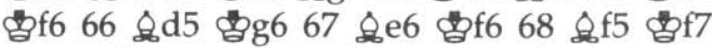

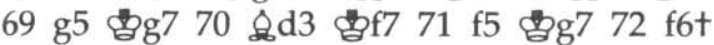

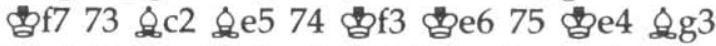
$76 \& d 1277 \& 4+1-0$ (adjudicated)

$$
\begin{gathered}
\text { BCP }- \text { Hitech } \\
\text { C45/08 } 11 \text { Bf4 }
\end{gathered}
$$

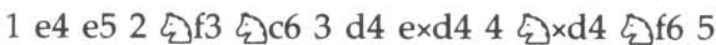

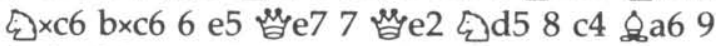

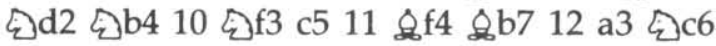
$13 \mathrm{O}-\mathrm{O}-\mathrm{O}$ h6 14 का

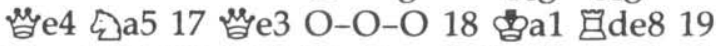

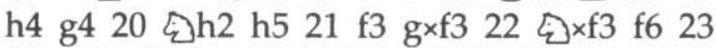

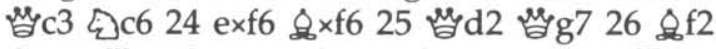

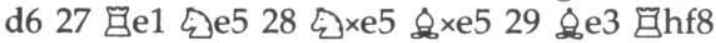

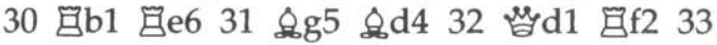
쌈 b3

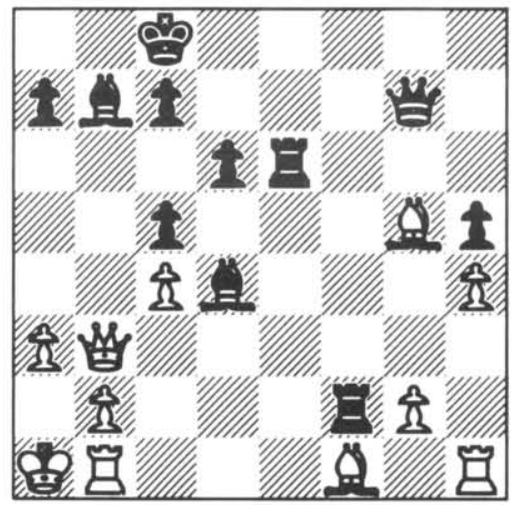

Position after 33 ․ㅏㅁ b3

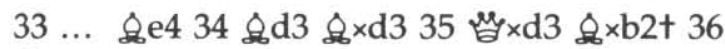

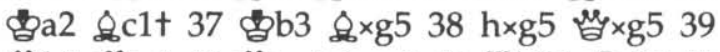

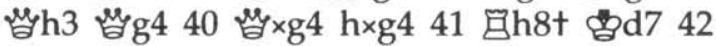

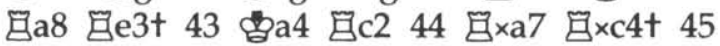

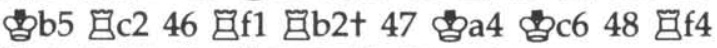
Ia2 49 日a6t 50 日×d6 c×d6 0-1

\section{Enterprise - Bobby A53/02 08 c5}

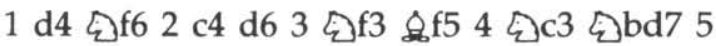
g3 e5 6 \&g2 c6 7 O-O h6 8 c5 e4 9 ¿d2

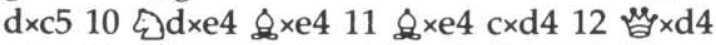

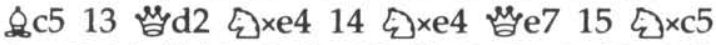

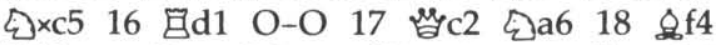

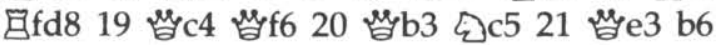

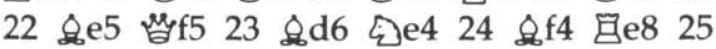




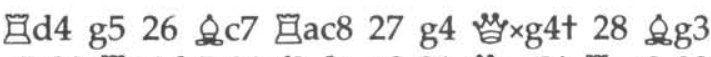

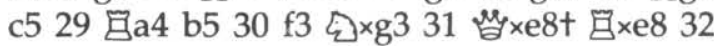

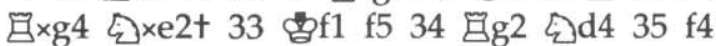
¿e6 $36 \mathrm{f} \times \mathrm{g} 5 \mathrm{~h} \times \mathrm{g} 5 \quad 37 \mathrm{~h} 4 \mathrm{~g} 4 \quad 38 \mathrm{~h} 5 \quad$ @ 4439

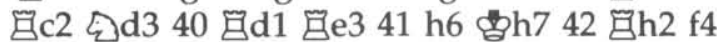
43 b3 c4 44 b×c4 b×c4 45 日h 4 c3 46 Ihh2 g3 47 日c2 f3 48 日b1 畐e6 0-1

\section{Mephisto - Dutch E92/01 10 Nd5}

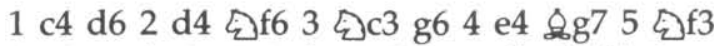

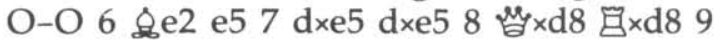

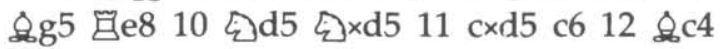

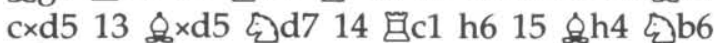

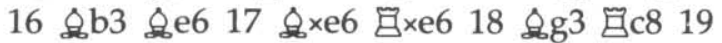

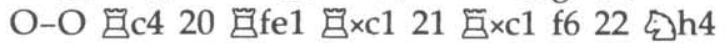

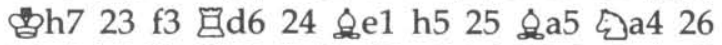

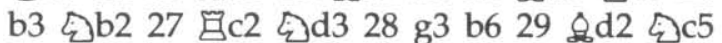

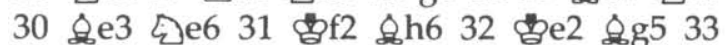

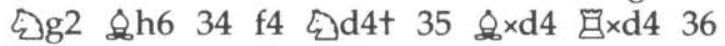

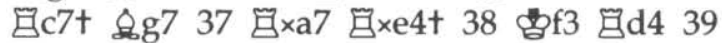

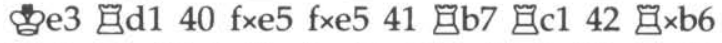

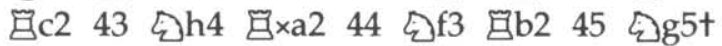

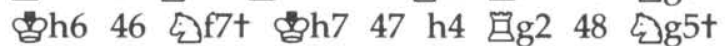

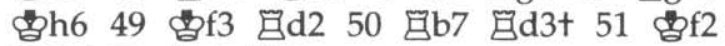

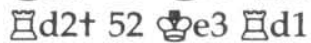

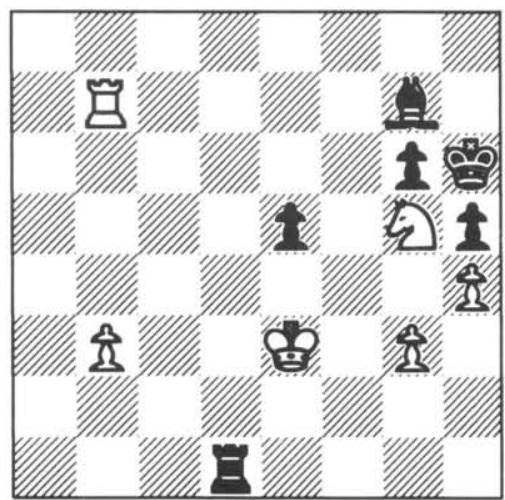

Position after $52 \ldots$ 目d1

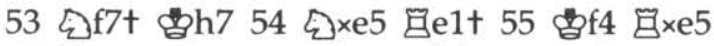

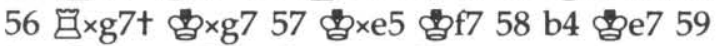

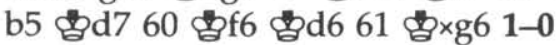

$$
\begin{gathered}
\text { Schach } 2.7 \text { - Nona } \\
\text { A52/13 06. Ng:e5 }
\end{gathered}
$$

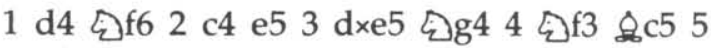

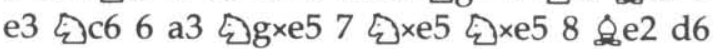
9 b4 \&b6 10 \&2 f6 11 O-O \&f5 12 a4 a5 13

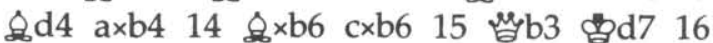

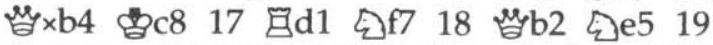

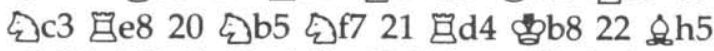

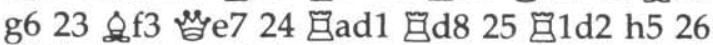

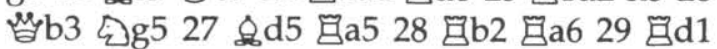

\&g4 30 日db1 1-0 (time)

$$
\begin{gathered}
\text { Rebel - Sun Phoenix } \\
\text { A44/04 04 Bd2 }
\end{gathered}
$$

$1 \mathrm{~d} 4$ c5 2 d5 e5 3 e4 d6 4 odd2 §f6 5 ¿c3 \&d7 6 \&e2 쌈66 7 घb1 ¿a6 8 \& 3 O-O-O

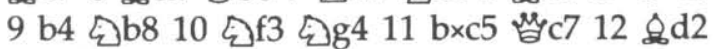

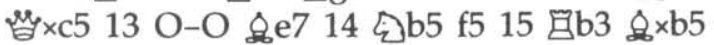

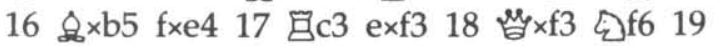

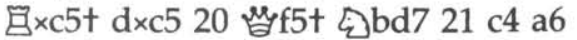

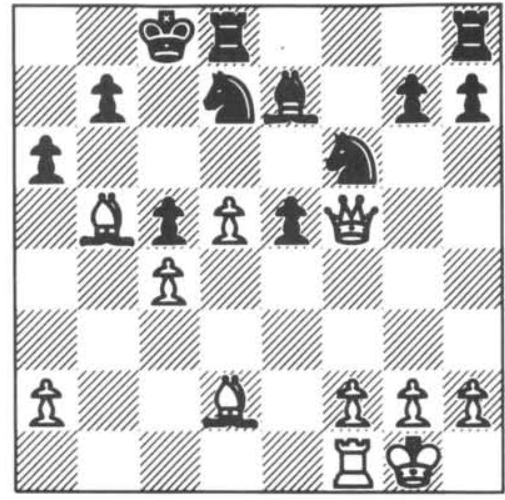

Position after $21 \ldots$ a6

$22 \& \times a 6$ § $2523 c \times d 5$ 1-

$$
\text { Cyrus 68K - Shess }
$$

C46/07 04 a3

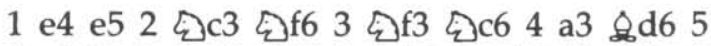
\& 4 O O-O 6 d3 a6 7 \&e3 b5 8 \&b3 쌈e7 9 a4

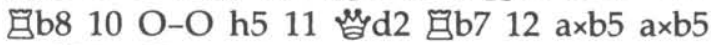

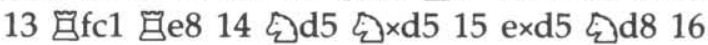
畐8

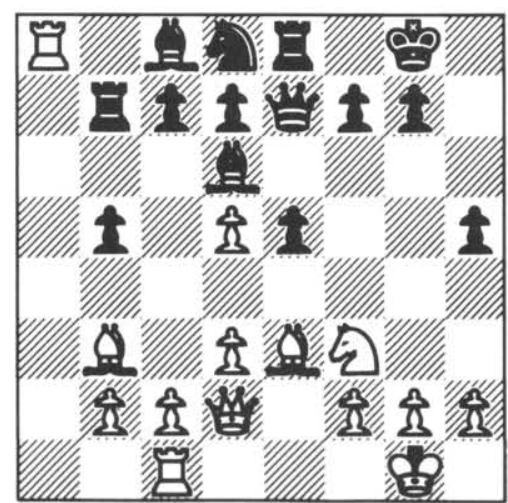

Position after 16 国

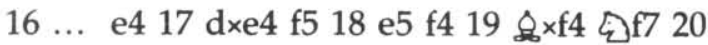

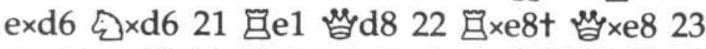

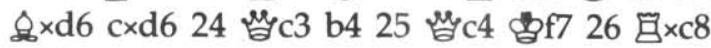
1-0 


\section{Round 2}

\author{
Hitech - Schach 2.7 \\ B86/01 $07 \mathrm{Be} 3$
}

1 e4 c5 2 ¿f 3 d6 3 \&c4 e6 4 d4 c×d4 5

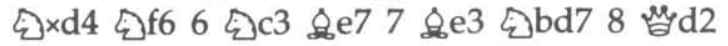

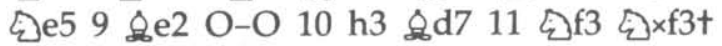

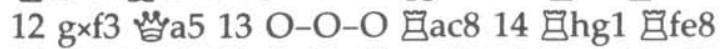

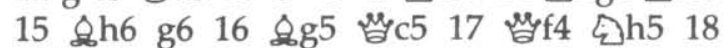
․ㅏㅁ $\mathrm{h} 4 \mathrm{f} 619$ \& $\mathrm{e} 3$ 쌈a5

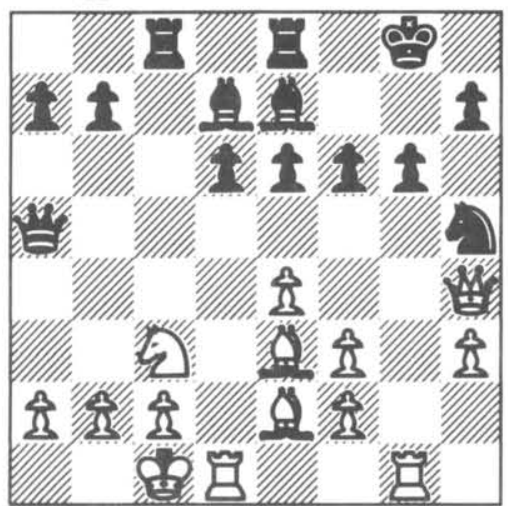

Position after 19 ... ․ㅏㅁa5

$20 \&$ b5 $\&$ b5 21 kㅏㅁh5 g5

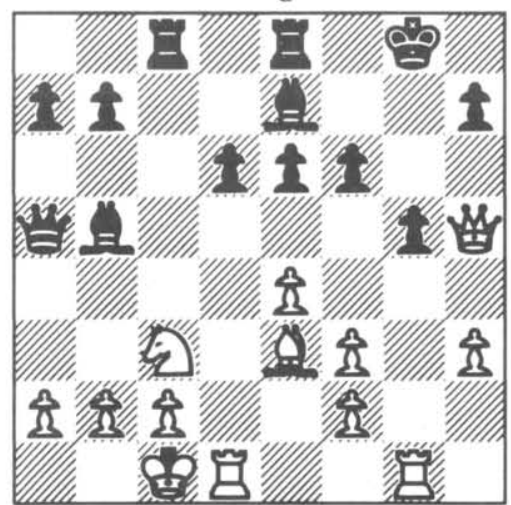

Position after 21 ... g5

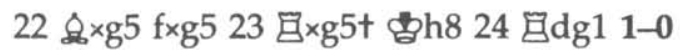

$$
\begin{gathered}
\text { Lachex - Rebel } \\
\text { C68/05 07. c5 }
\end{gathered}
$$

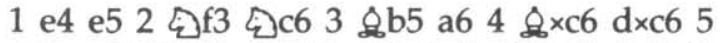

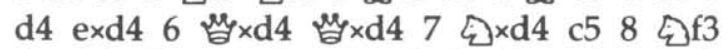
\&g4 9 \&f4 \&xf3 $10 \mathrm{~g} \times \mathrm{f3} \quad \mathrm{O}-\mathrm{O}-\mathrm{O} 11 \mathrm{O}-\mathrm{O}$ \&d6 12 e5 \&e7 13 §c3 f6 14 Gad1 fxe5 15

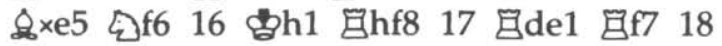

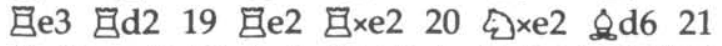

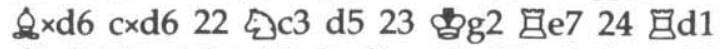

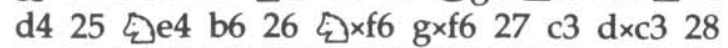

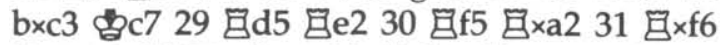

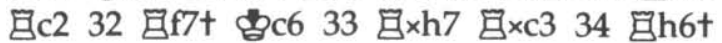

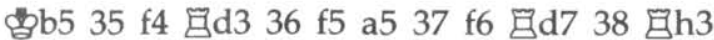

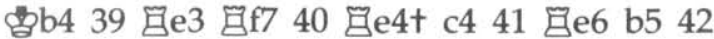

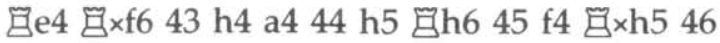
包2 c3 47 f5 0-1

$$
\begin{gathered}
\text { Dutch - Enterprise } \\
\text { A07/04 05. e5 }
\end{gathered}
$$

1 ¿f3 d5 2 g3 c6 3 \&g2 \&g4 4 O-O 乞d7 5 d3 e5 6 ¿bd2 \&c5 7 h3 \&f5 8 c3 f6 9 d4

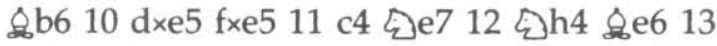
cxd5 c×d5 14 ¿b3 O-O 15 \&g5 @f6 16 Ilc1

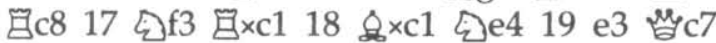

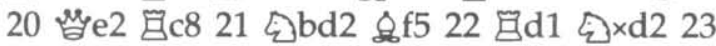

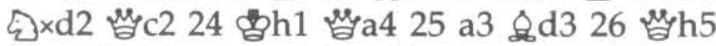

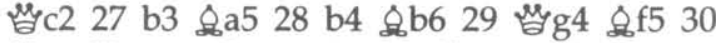

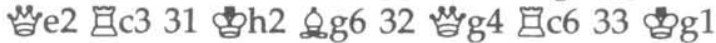

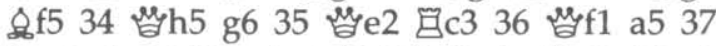

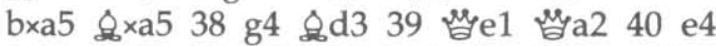

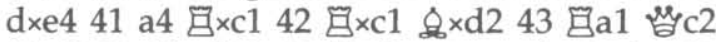

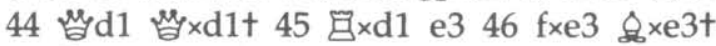

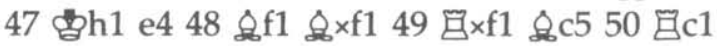
b6 51 a5 e3 52 gig2 \&d4 53 a6 b5 54 a7

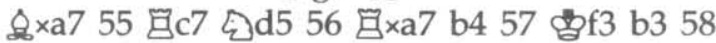

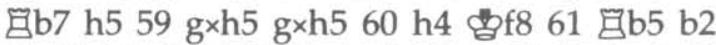

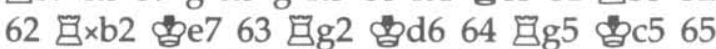

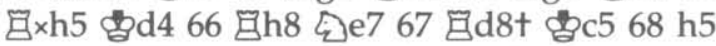

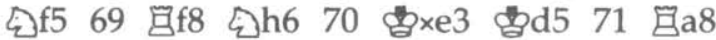

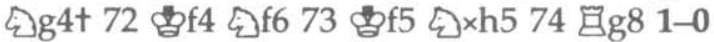

$$
\begin{gathered}
\text { Awit - Ostrich } \\
\text { A21/12 05. Ne7 }
\end{gathered}
$$

1 c4 e5 2 Ðc3 d6 3 g3 g6 4 \&g2 \&g7 5 d3

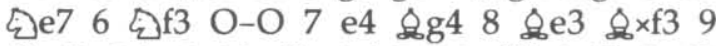

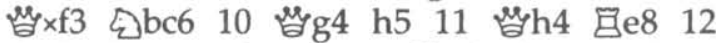

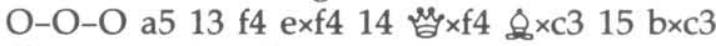

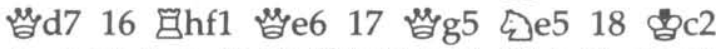
¿g4 19 \&g1 f6 20 쌈b5 b6 21 Gb1 Gad8 22 d4 f5 23 gfe1 c6 24 exf5 쌈 xe1 25 Gxe1 c×b5

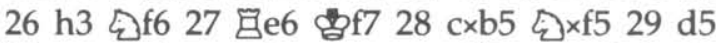
Gxe6 30 dxe6t

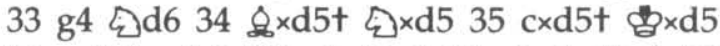

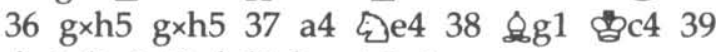
\&e3 $气$ c3 40 \&d2

$$
\begin{gathered}
\text { Plymate - Mephisto } \\
\text { B18/10 09 N:g6 }
\end{gathered}
$$

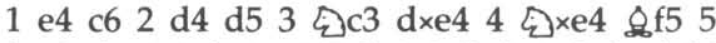

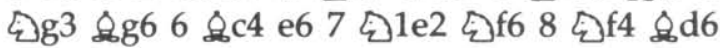

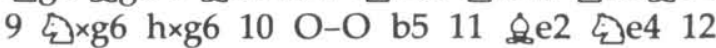

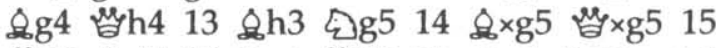

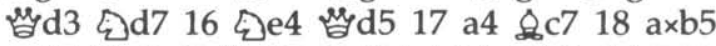

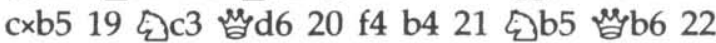

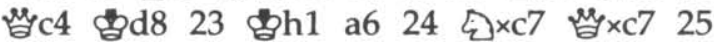


쌈b3 쌈c6

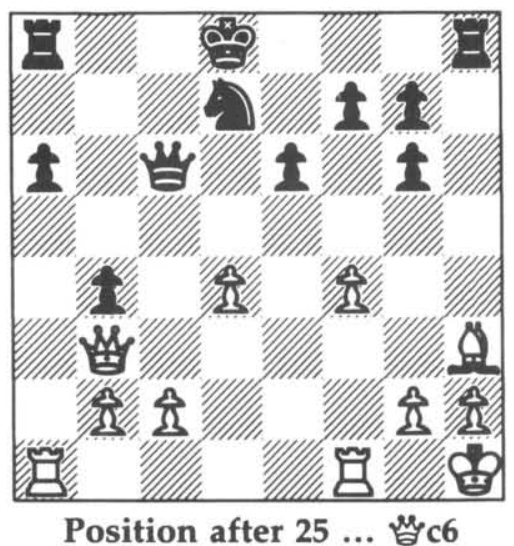

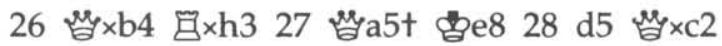

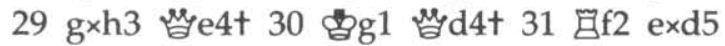

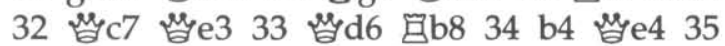

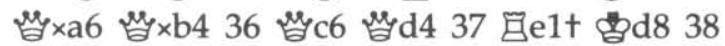

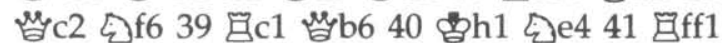

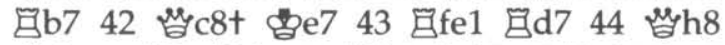

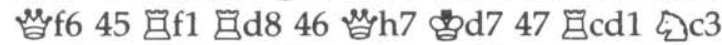

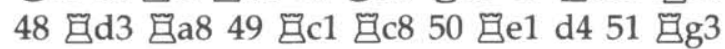

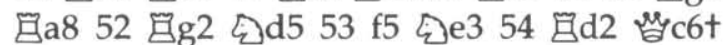

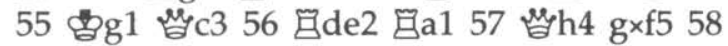

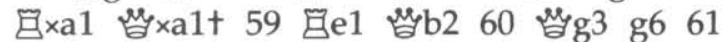

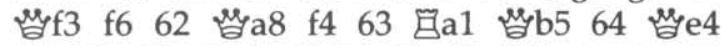

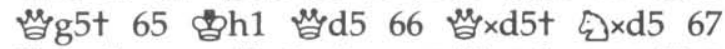

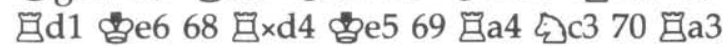
¿d5 71 by

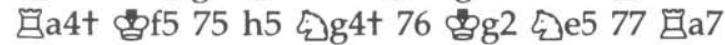

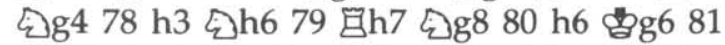
星g7t

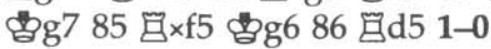

\section{Kempelen - Vaxchess B02/01 $02 \mathrm{~d} 4$}

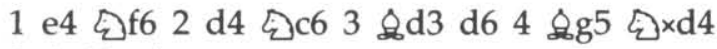
0-1 (time)

\section{Sun Phoenix - Advance 68K A45/09 03. d5}

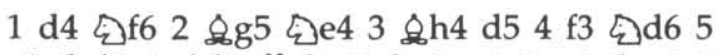

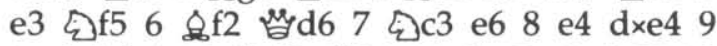

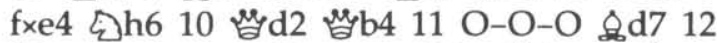

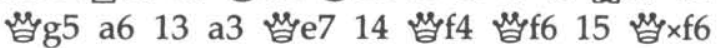

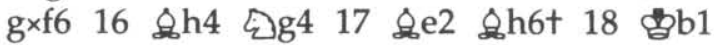

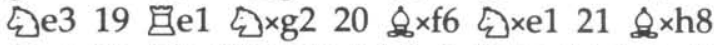

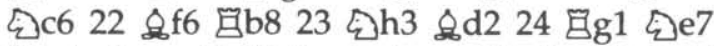

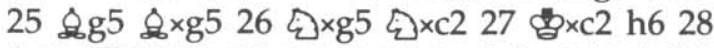

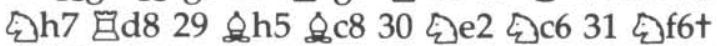

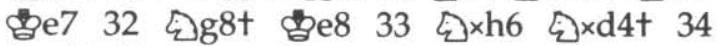

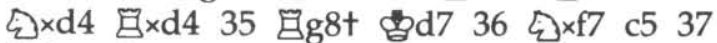

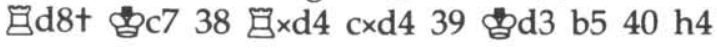

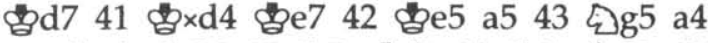

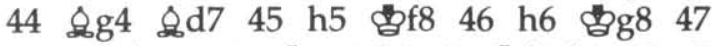

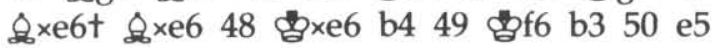
夏h8 51 e6 1-0

$$
\begin{gathered}
\text { Chat - Rex } \\
\text { A58/04 06. e5 }
\end{gathered}
$$

1 d4 2 f6 2 c4 c5 3 d5 b5 4 c×b5 a6 5 b×a6

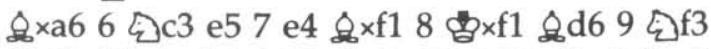

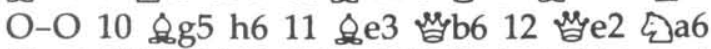
13 a4 回fb8 14 日a2 §b4 15 日a1 g5 16 h4 g4

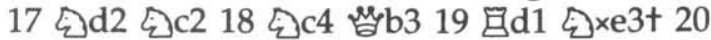

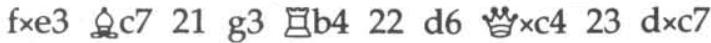

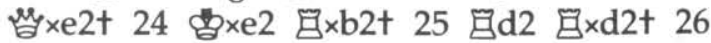

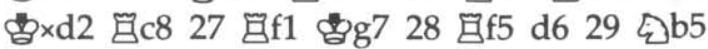

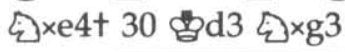

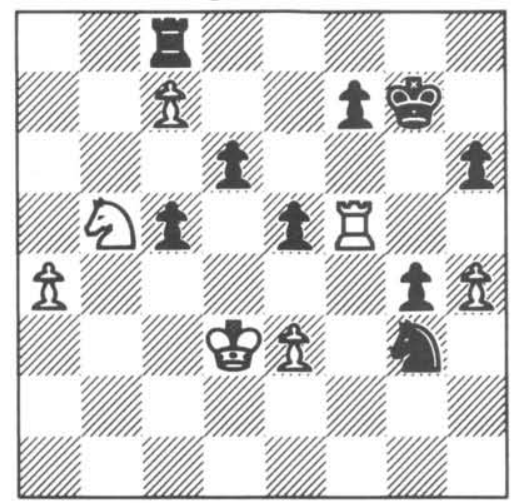

Position after $30 \ldots$... $2 \times \mathrm{g} 3$

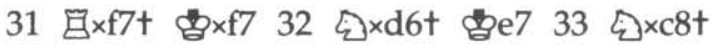

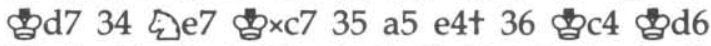

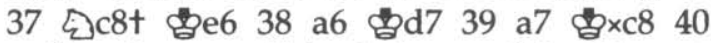

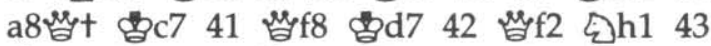

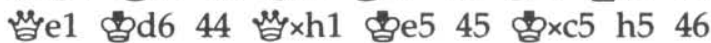

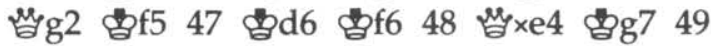

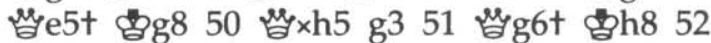
e4 g2 53 e5 g1쌈 54 쌈 $\times$ ( 1-0

$$
\begin{aligned}
& \text { Nona - BCP } \\
& \text { D96/01 05. e6 }
\end{aligned}
$$

1 d4 $乞 f 62$ c4 g6 3 ¿c3 d5 4 ¿f3 \&g7 5

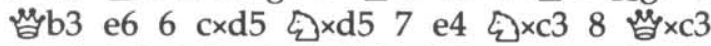
O-O 9 \&f4

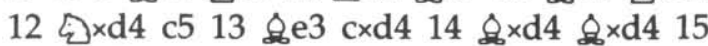

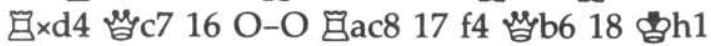

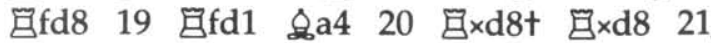

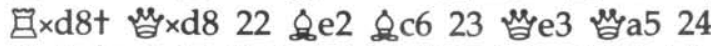

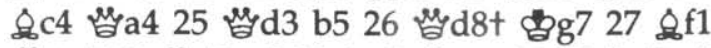

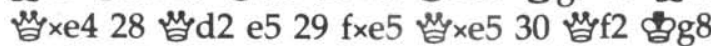

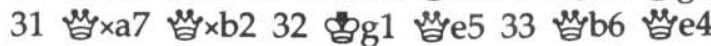

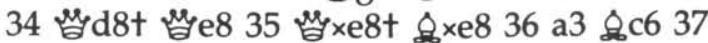
कीf2 h5 38 का 
41 bb5 f4 42 a4 54 a5 43 a 84 a6

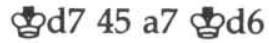

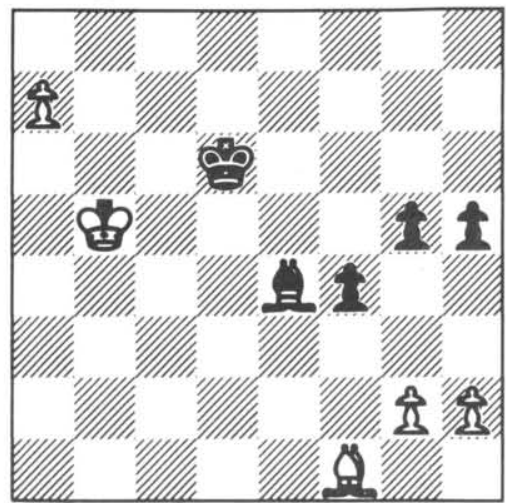

Position after 45 ...

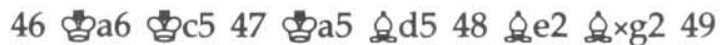
\&b5 \&d5 50 \&d3 f3 51 \&f 1 f2 52 \&e2 \&e4 53 \&b5 \&f3 54 \&d3 $\quad$ कd 55 \&b5 क्षुc3 56

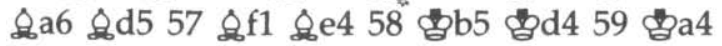
bic5 60 ba h4 61 h3 \&d5 62 ba 63

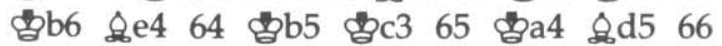

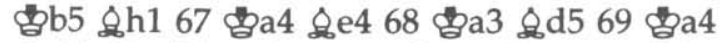

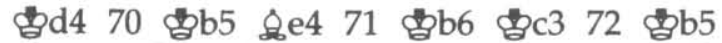

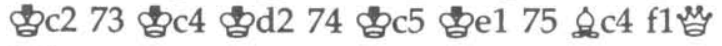
0-1

\section{Bebe - Cyrus 68K $\mathrm{B} 00 / 02$ 02. e6}

1 e4 $乞$ c6 2 d4 e6 3 d5 exd5 4 exd5 $气$ e5 5

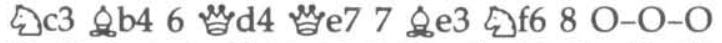

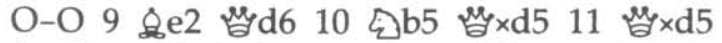

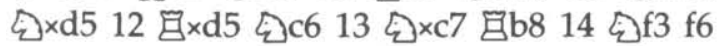
15 a3 $2 \mathrm{a} 5$

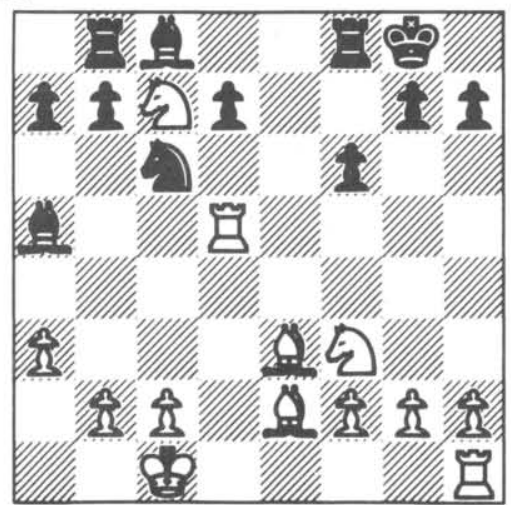

Position after 15 ... \& a5

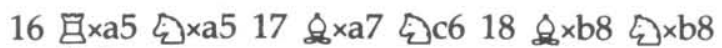
19 回d1 1-0

\section{Bobby - Cray Blitz C29/09 12 c3}

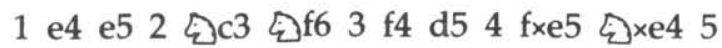

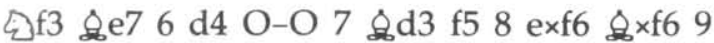

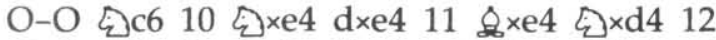

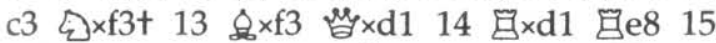

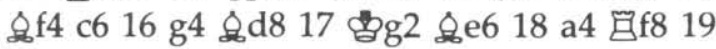

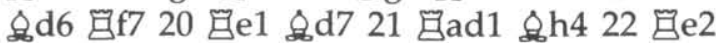

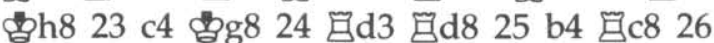
Iee3 \&g5 27 Ie1 目d8 28 \&c5 b6 29 \&d6 \&h4 30 百e5 \&f6 31 目e2 \&g5 32 h3 国 833 a5 b×a5 34 目5 $\quad 8 \mathrm{~d} 835 \mathrm{~b} \times \mathrm{a} 5$ \&f6 36 目e1 \&d8 37 a6 \&h4 38 每e2 目d8 39 \&c5 \&g5 40

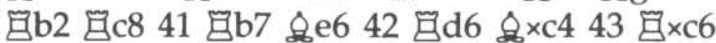

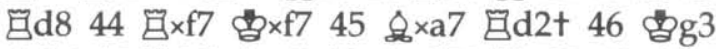

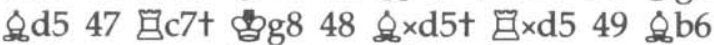

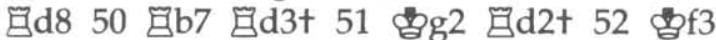

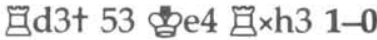

\section{Round 3}

\section{Plymate - Hitech C78/11 $07 \mathrm{~d} 3$}

1 e4 e5 2 ¿f3 2 c6 3 \&b5 a6 4 \&a4 2 f6 5 O-O b5 6 b3 \&b7 7 d3 \&c5 8 \&c3 O-O 9

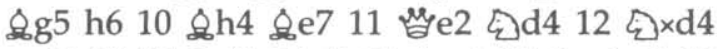
exd4 13 @b1 d5 14 @d2 dxe4 15 dxe4 c5 16

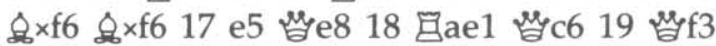

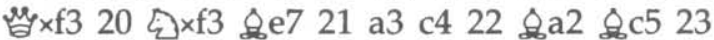

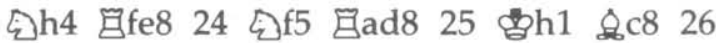
气े 3

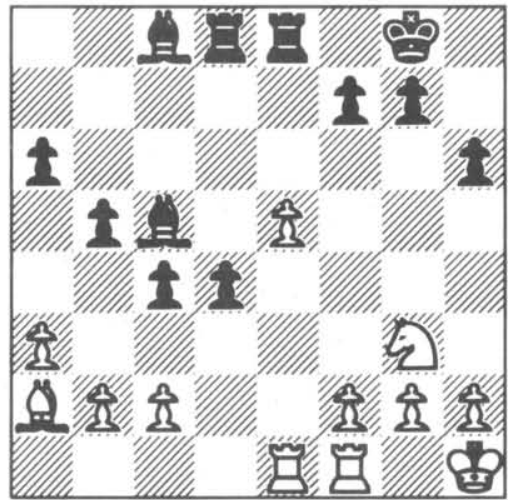

Position after 26 g3

$26 \ldots$ d3 $27 \quad c \times d 3 \quad c \times d 328$ f4 d2 29 日b1 $8 \mathrm{e} 3$ 30 \&b3 \&e6 31 \&c2 目e7 32 \&d1 目c7 33 f5 \&c4 34 \&e2 月e7 35 e6 f×e6 36 f×e6 目×e6 37

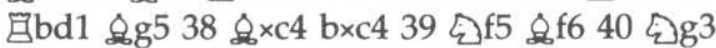
Q $\times b 2$ 0-1

$$
\begin{gathered}
\text { Rebel - Bobby } \\
\text { A53/02 } 08 \text { d5 }
\end{gathered}
$$

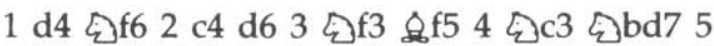
g3 e5 6 \&g2 c6 7 O-O h6 8 d5 c×d5 9 Ø×d5

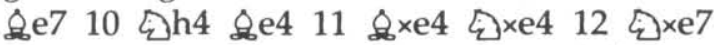

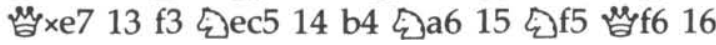




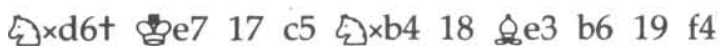

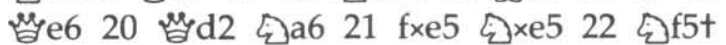

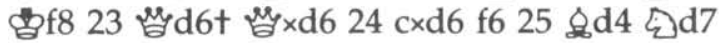

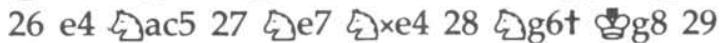

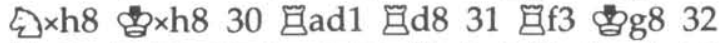

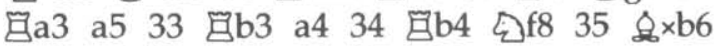

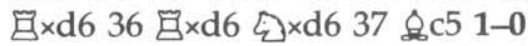

$$
\begin{gathered}
\text { Mephisto - Chat } \\
\text { A28/08 07. Bd7 }
\end{gathered}
$$

1 c4 e5 2 ¿c3 ¿f6 3 ¿f3 ¿c6 4 e3 d5 5

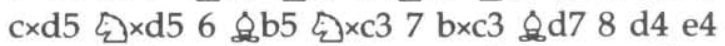

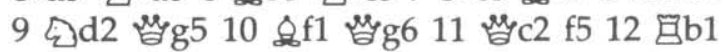
b6 13 g3 \&e7 14 \&e2 O-O 15 씸b3t \&e6 16

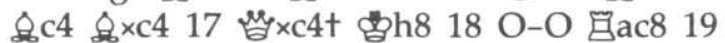

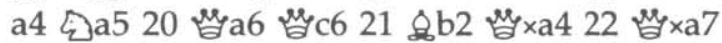

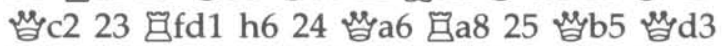

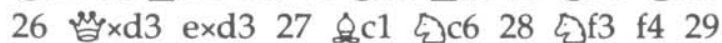
exf4 冒a2 30 品d3 自fa8 31 d5

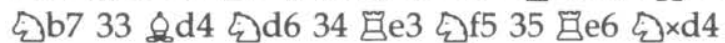
36 乞xd4 \&c5 37 冒c6 \&xd4 38 cxd4 回8a7 39 d6 号a1

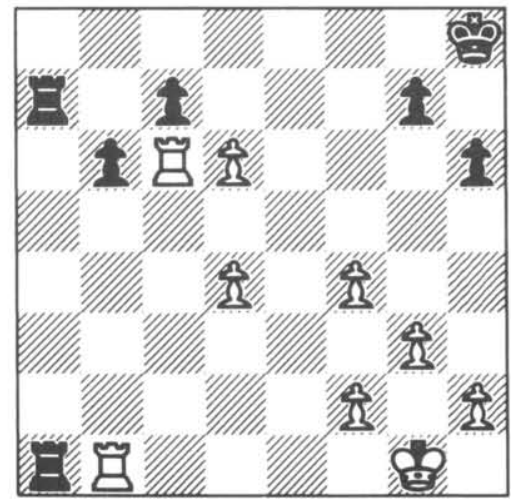

Position after $39 \ldots$... 国1

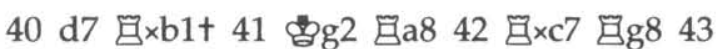

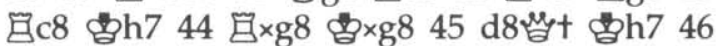
d5 b5 47 d6 b4 48 당 8 目a1 49 d7 冒d1 50

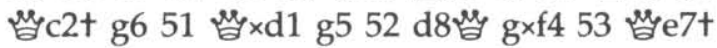
1-0

\section{Cyrus 68K - Ostrich B29/04 04. N:d4}

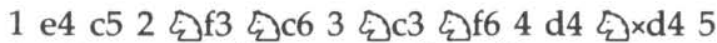

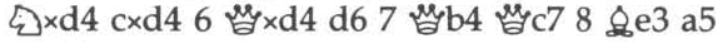
9 씩a4t \&d7 10 \&b5 O-O-O 11 \& $2 \times d 7 t$

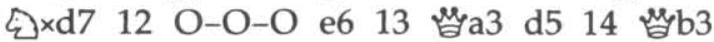

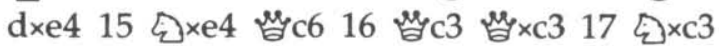
\&b4 18 ¿e4 f5 19 ¿d6t \&xd6 20 Gxd6 国he8 21 \&d2 22 月d3 月a8 23 月g3 g6

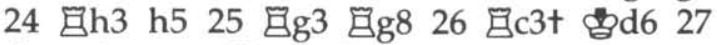

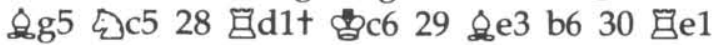
bd6 31 \&xc5t bxc5 32 冒ce3 冒ae8 33 月a3
目8 34 冒d3十

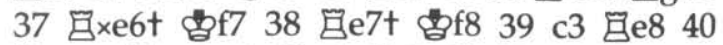

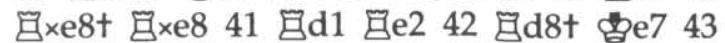

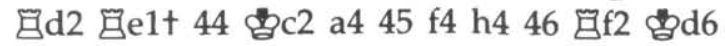

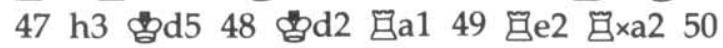

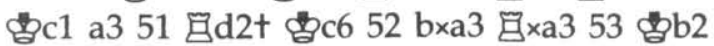

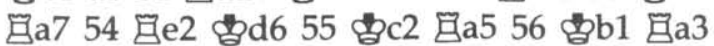

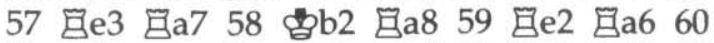

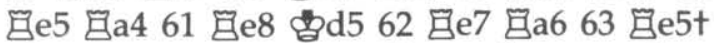

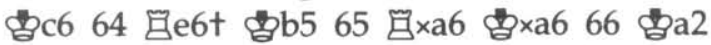

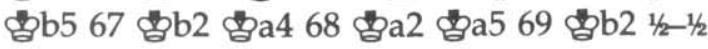

$$
\begin{gathered}
\text { Cray Blitz - Bebe } \\
\text { B70/05 10. Nd7 }
\end{gathered}
$$

1 e4 c5 2 ¿f3 d6 3 d4 cxd4 4 ¿xd4 ¿f6 5 ¿c3 g6 6 \&e2 \&g7 7 O-O O-O 8 \&g5 乞c6 9 ¿b3 \&e6 $10 \mathrm{f} 4$ ¿d7 11 씸d3 h6 12 \&h4

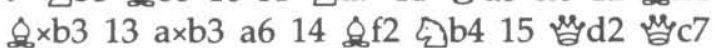

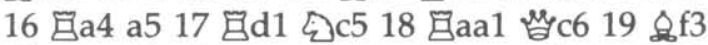

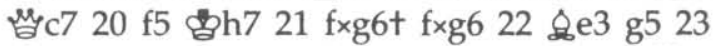

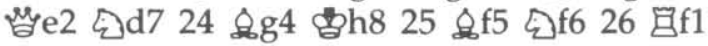

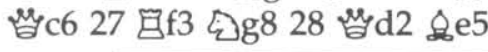

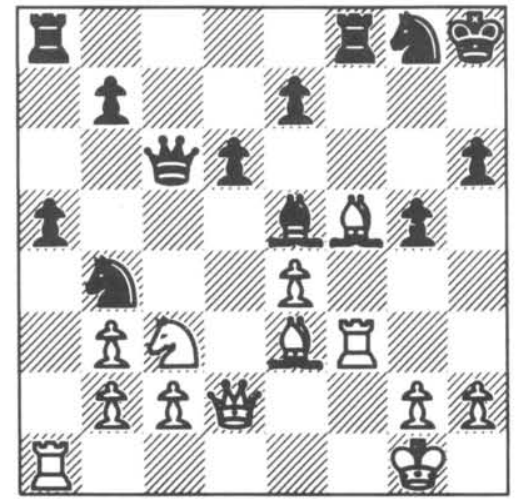

Position after $28 \ldots$...

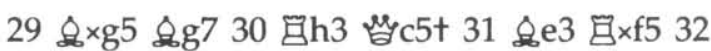

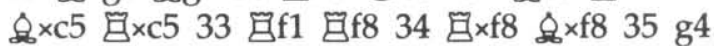

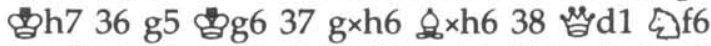

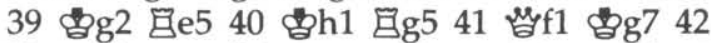

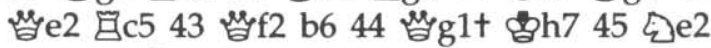
¿ेh5 46 쌈 4 1-0

\section{Advance 68K - Dutch B09/32 07. e5}

1 e4 d6 2 d4 §f6 3 ¿c3 g6 4 f4 \&g7 5 \&f3 O-O 6 \&d3 2 c6 7 O-O e5 8 dxe5 dxe5 9 f5 gxf5 10 exf5 e4 $11 \quad \xi \times e 4 \quad \& \times f 5 \quad 12 \quad$ ¿ $\times f 6 t$

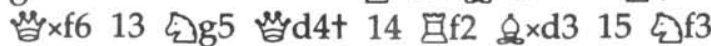

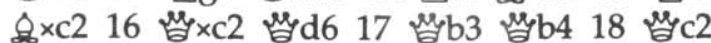

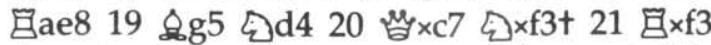

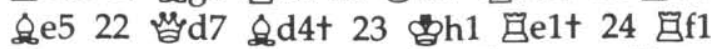

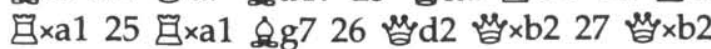

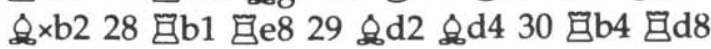


31 日a4 h5 32 g3 b5 33 Ga6 \&e5 34 \&e3 h4

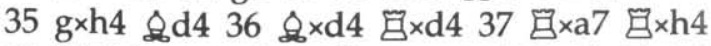

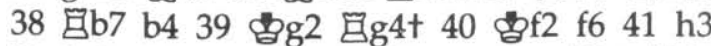

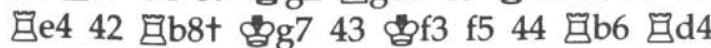

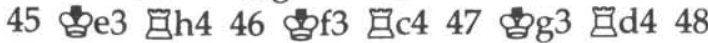

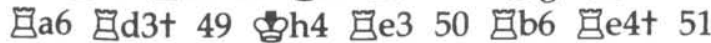

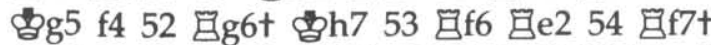

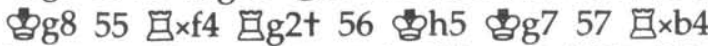

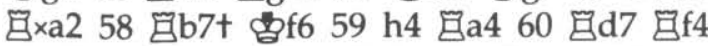

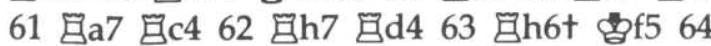

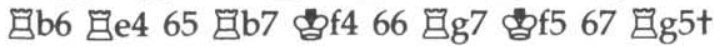
कोf6 68 日g4

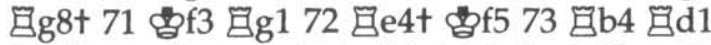

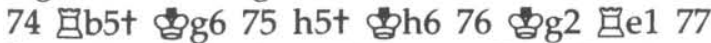

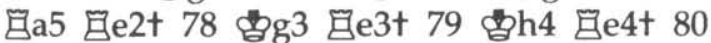

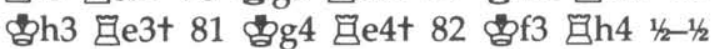
(adjudicated)

\section{BCP - Sun Phoenix $\mathrm{C} 02 / 0606 \mathrm{Bd} 3$}

1 e4 e6 2 d4 d5 3 e5 c5 4 c3 ․ㅏㅁb6 5 @f3

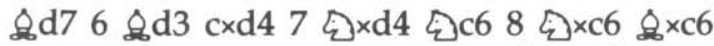
9 O-O O-O-O 10 气d2 f6 11 ㅆgg4 冒e8 12

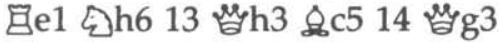

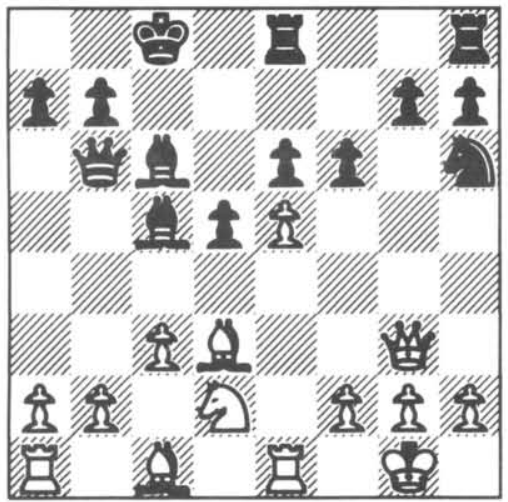

Position after 14 kgh 3

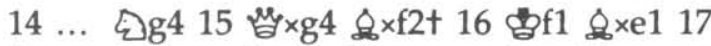
क्

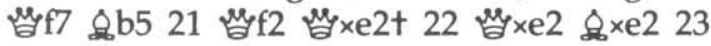

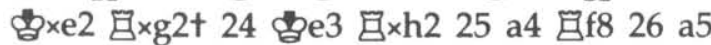

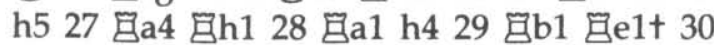

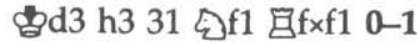

\section{Vaxchess - Lachex D52/11 10 Bf4}

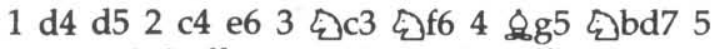

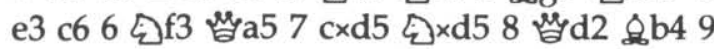
Ilc1 h6 10 \&f4 O-O 11 \&g c5 12 \&d3 f5 13 $\mathrm{O}-\mathrm{O}$ cxd4 14 exd4 f4 15 쌈e1 $§ 7 \mathrm{f} 616$ \&h4

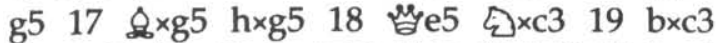

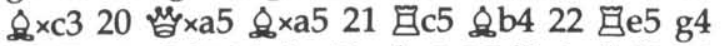

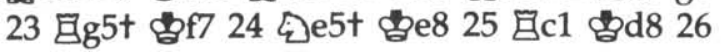

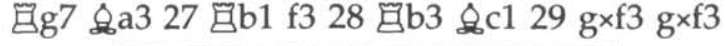

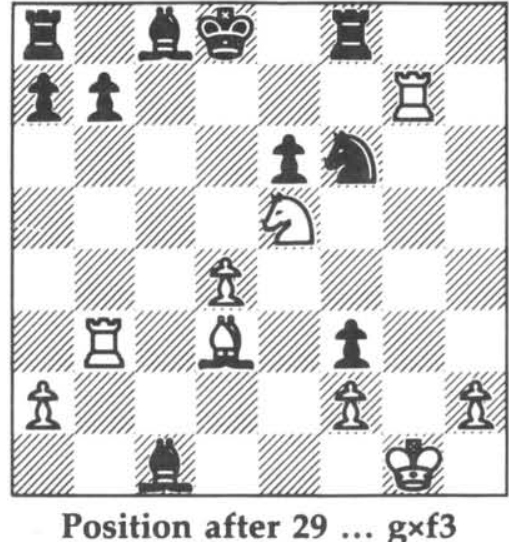

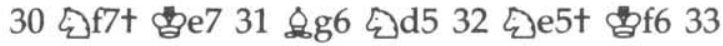

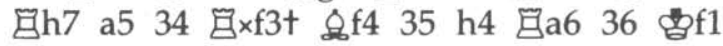

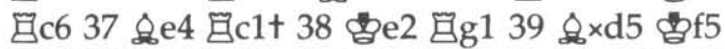
40 日h5t dff6 41 \&e4 42 §d3 \&d6 43 目×f8 \&a6 47 a4 e5 48 dxe5 $8 \times e 549$ bुe3 \& 750

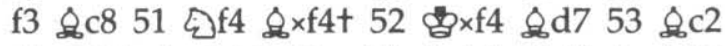
b7 54 b5 b5 55 axb5 \& \&b5 56 f4 \&c4 57

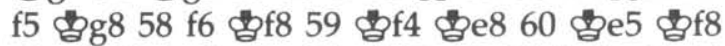

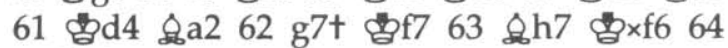

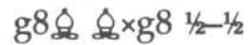

\section{Enterprise - Awit D87/04 10 d5}

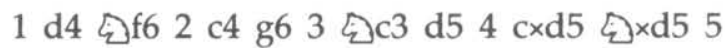
e4 $气 \times c 36$ bxc3 c5 7 \&c4 \&g7 8 乞e2 O-O 9

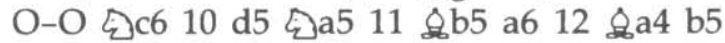

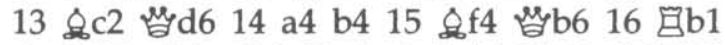

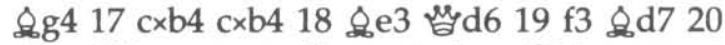

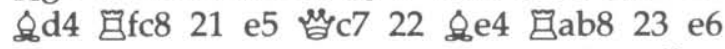

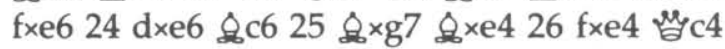

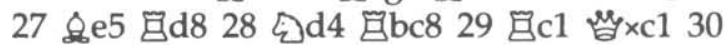

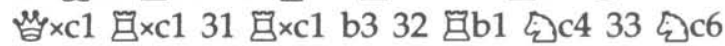

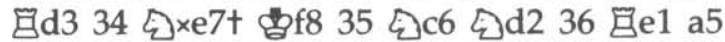

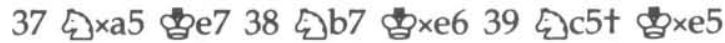
40 ¿xd3t

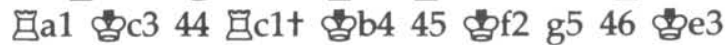

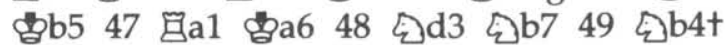

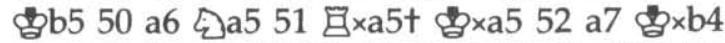

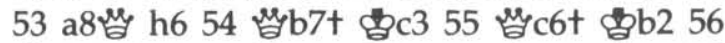

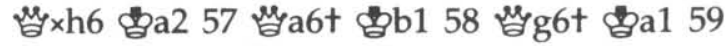

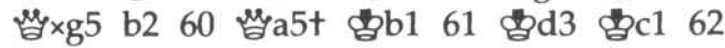
․ㅏㅁe1\# 1-0

$$
\begin{gathered}
\text { Rex - Nona } \\
\text { C73/02 07. Nf6 }
\end{gathered}
$$

1 e4 e5 2 ¿f3 2 c6 3 \&b5 a6 4 \&a4 d6 5

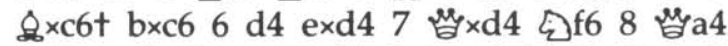

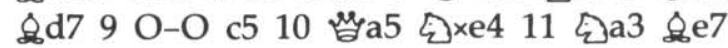


12 包1 f5 13 \&f4 O-O 14 ¿c4 \&b5 15

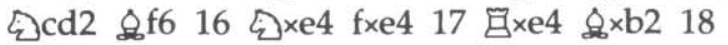

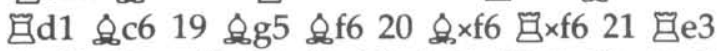

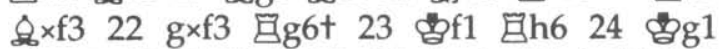

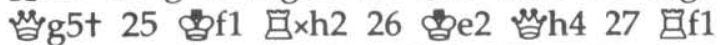

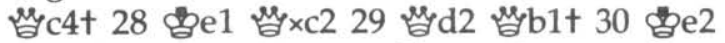

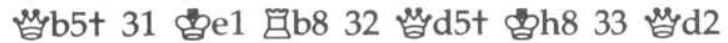

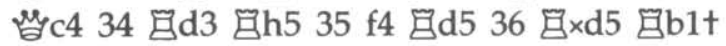

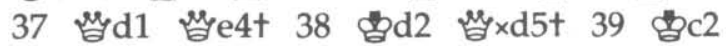

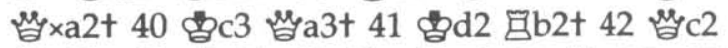

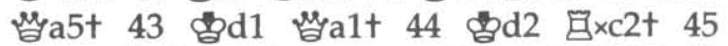

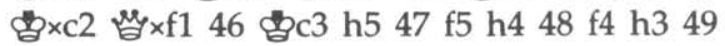
f6 $\mathrm{g} \times \mathrm{f6} 0-1$

\section{Schach 2.7 - Shess \\ D59/12 13 Be2}

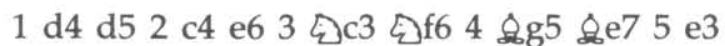

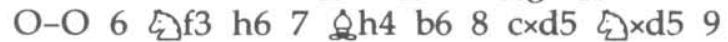

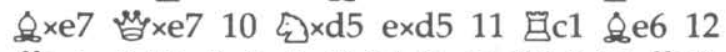

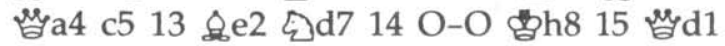
Gae8 16 b5 冒d8 17 目e1 g5 18 kㅏㅁa4 g4 19

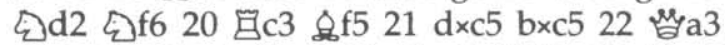

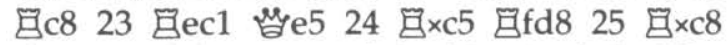

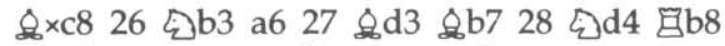

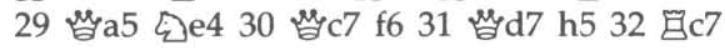
\&cc6 33 쌈 $\mathrm{h} 7 \#$ 1-0

\section{Round 4}

$$
\begin{gathered}
\text { Awit - Rex } \\
\text { A01/21 04. Qg5 }
\end{gathered}
$$

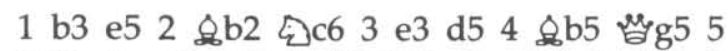

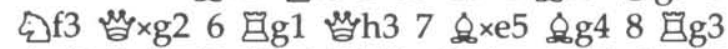

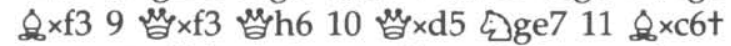

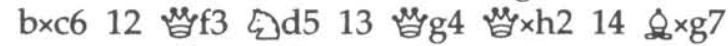

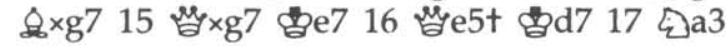

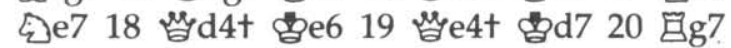

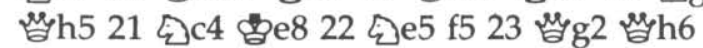

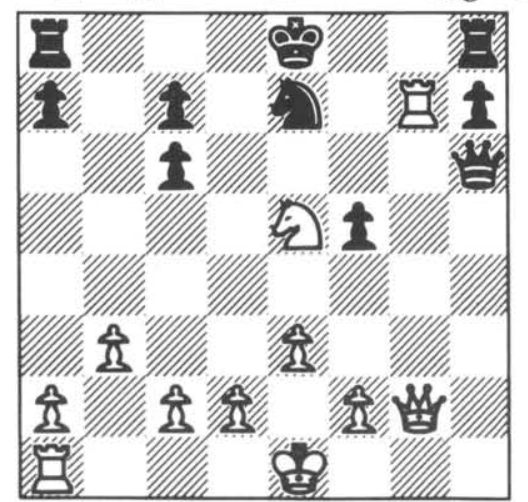

Position after 23 ... 츰h6

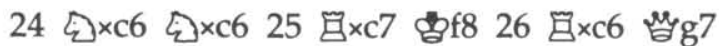

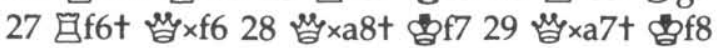

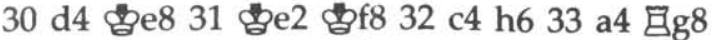

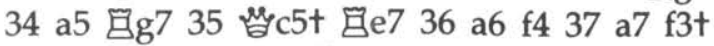

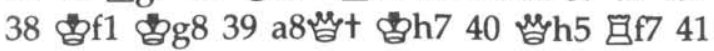

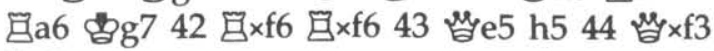
1-0

$$
\begin{aligned}
& \text { Lachex - BCP } \\
& \text { B22/07 07 Na3 }
\end{aligned}
$$

1 e4 c5 2 c3 $\leftrightharpoons$ f6 3 e5 $\leftrightharpoons d 54$ d4 c×d4 5 cxd4

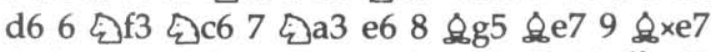

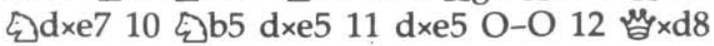

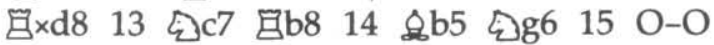

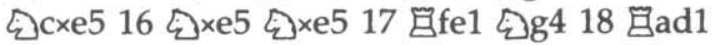

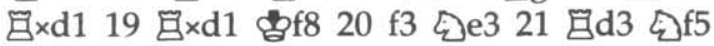

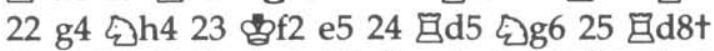

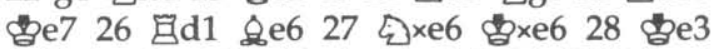

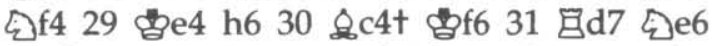
32 tge3 a5 33 a4 g6 34 h4 b6 35 b3 g5 36 h5

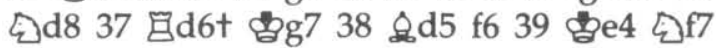

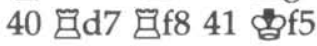

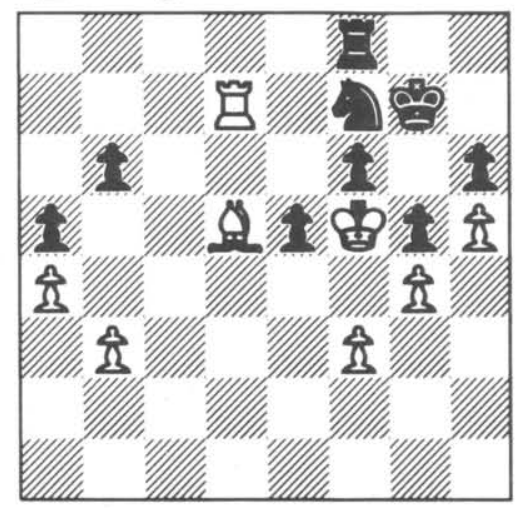

Position after 41 d 5

41 ... e4 42 f×e4 b5 43 a×b5 a4 44 b×a4 4 th 8 45 \& $\times f 7$ 目c8 46 b 目d8 f×e4 49 \&h7\# 1-0

\section{Chat - Enterprise E50/04 07 Bd2}

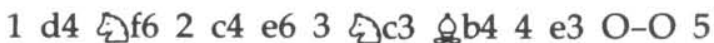
\&d3 c5 6 ¿f 3 ¿c6 7 \&d2 cxd4 8 exd4 d5 9

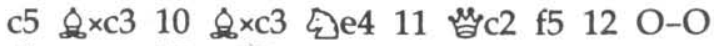

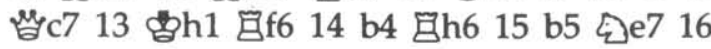

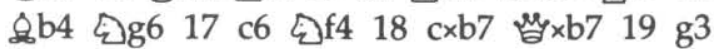

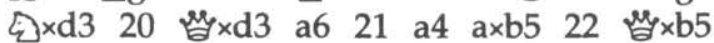

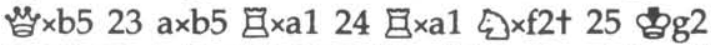

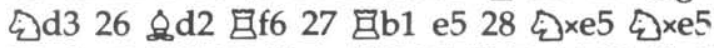

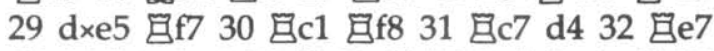
d3 33 b6 \&a6 34 国a7 \&b5 35 gf 8 \&c6t 36

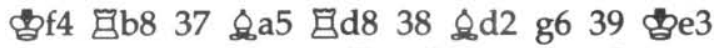

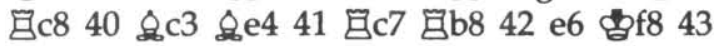
\&g7t tre8 44 \&d4 h6 45 Ig7 d2 46 घg8t

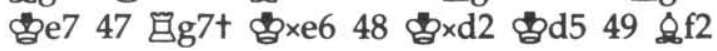




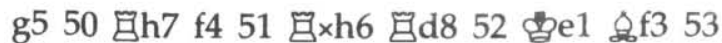

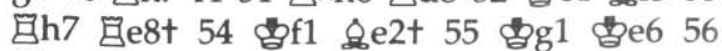

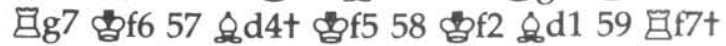

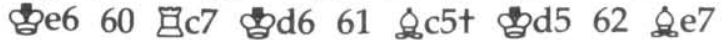

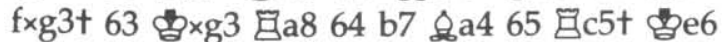

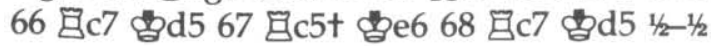

\section{Bobby - Mephisto B03/03 08. Nc6}

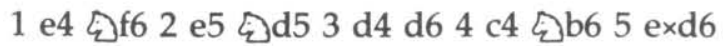

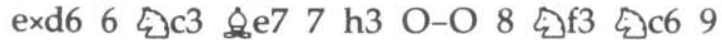
\&e2 \&f5 10 O-O 쌈d7 11 \&f 4 Iae8 $12 \mathrm{a} 4$ \&f6 13 a5 2 c8 14 a6 b6 15 g4 \& $\times 9416$ h×g4

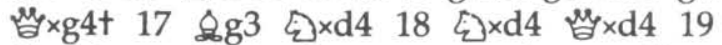

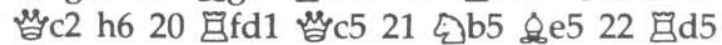

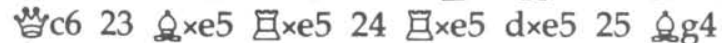

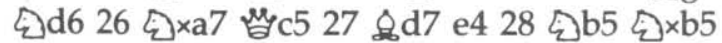

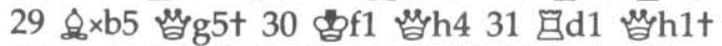

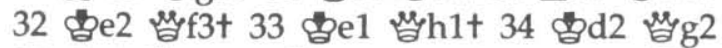

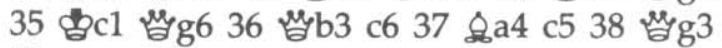

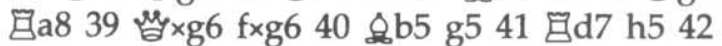
a7 e3 43 fxe3 目f8 44 \& 86 h4 45 a8 쌈 目×a8 46 \& $\times a 8$ h3 47 日d5 1-0

\section{Hitech - Rebel C33/08 03. Qh4+}

1 e4 e5 2 f4 exf4 3 \&e2 쌈h4t 4 要f1 2 f6 5 ¿c3 $\& 46$ e5

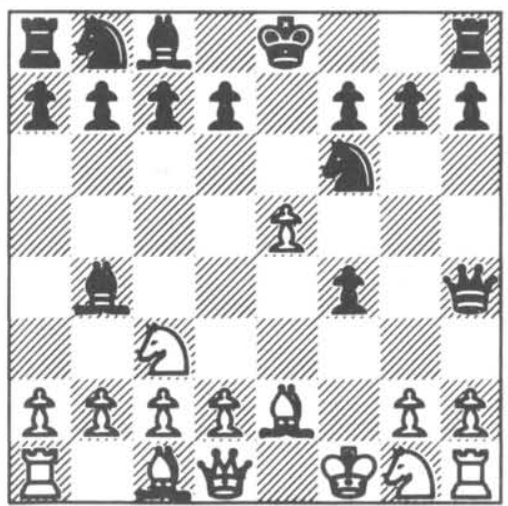

Position after 6 e5

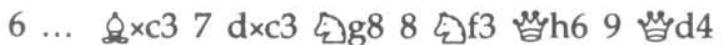

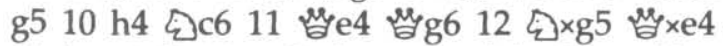
13 ¿xe4 f3 14 g×f3

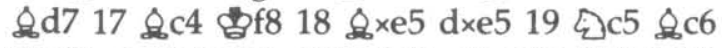

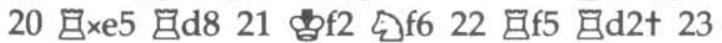

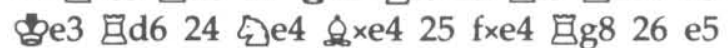
Ic6 27 exf6 1-0

\section{Bebe - Advance 68K B74/01 09. Bd7}

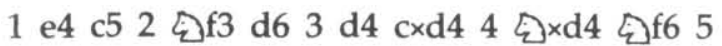

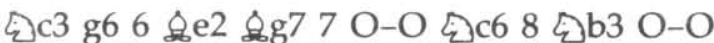
9 \& 3 \&d7 $10 \mathrm{f} 4$ \&e6 11 f5 g×f5 12 exf5 \&d7

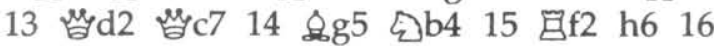

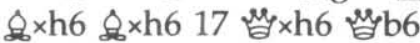

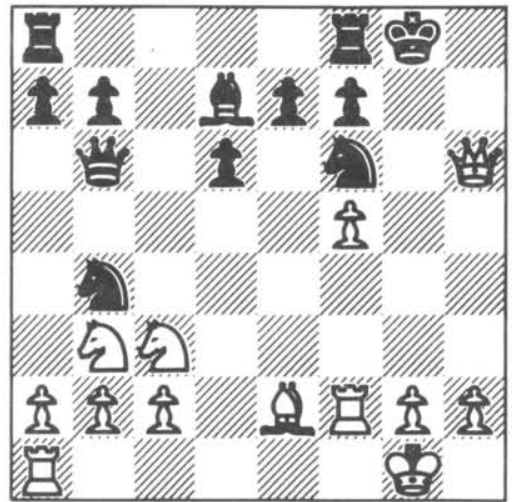

Position after 17 ... ․ㅏㅁb6

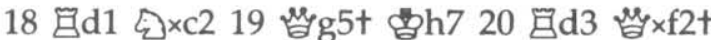

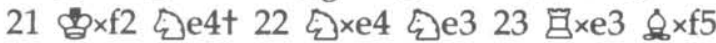

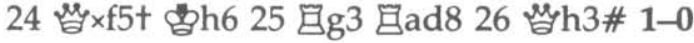

\section{Sun Phoenix - Plymate D00/05 05 c4}

1 d4 d5 2 \&g5 2 f6 3 \& xf6 exf6 4 e3 c6 5 c4

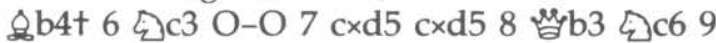

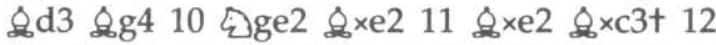

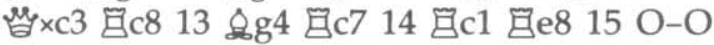
छee7 16 쌉d2 쌈d6 17 f3 b5 18 国5 a6 19

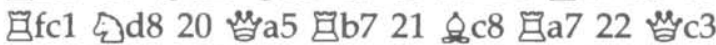

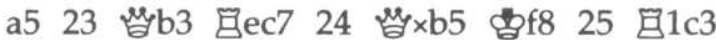

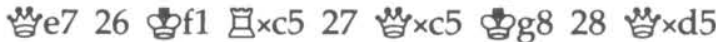

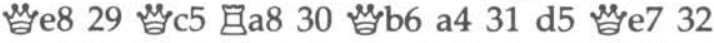
d6 쌈f8 33 \&g4 a3 34 bxa3 쌈e8 35 d7 ․ㅏㅁe7 36 日 $\mathrm{c} 8$ 쌈 $\times \mathrm{d} 7$ 1-0

\section{Ostrich - Vaxchess C68/05 09 O-O}

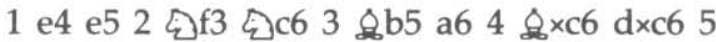

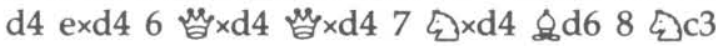
¿e7 9 O-O h5 10 国1 \&g4 11 e5 \&b4 12 f3

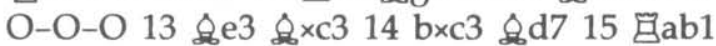

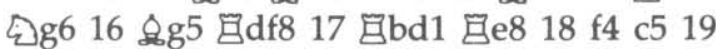
¿f 3 \&g4 20 日f 1 h4 21 h3 $8 \mathrm{f} 5 \quad 22$ 日f2

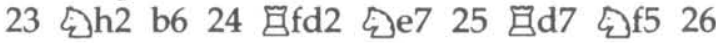
畐 $\times$ 77 1-0

\section{Shess - Nona \\ C43/15 09 Bd3}

1 e4 e5 $2 \succeq f 3$ f6 3 d4 exd4 4 e5 $§$ e4 5

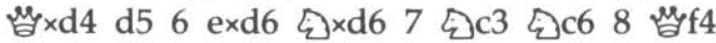

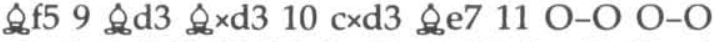

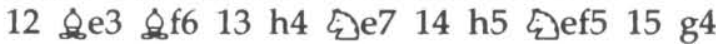




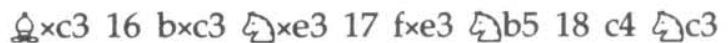

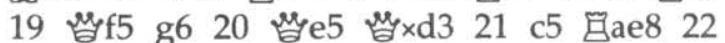

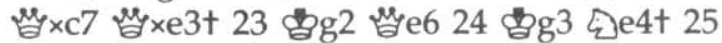

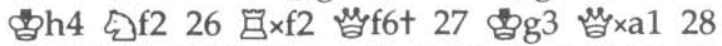

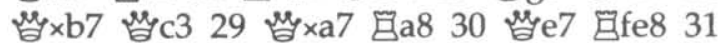

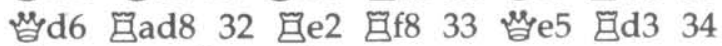

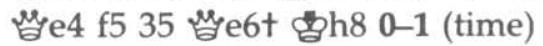

\section{Dutch - Cyrus 68K D02/04 03. Nf6}

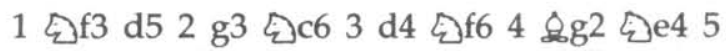

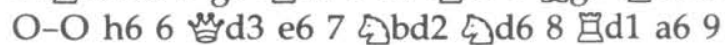
c4 ¿f5 10 cxd5 exd5 11 e3 \&e7 12 ¿e5

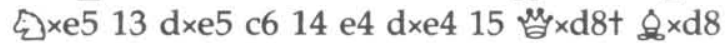
16 ¿xe4 O-O 17 b3 \&b6 18 \&b2 \&e6 19

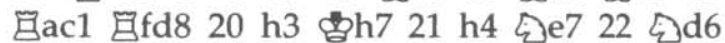

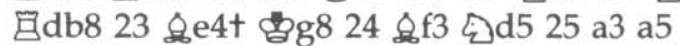

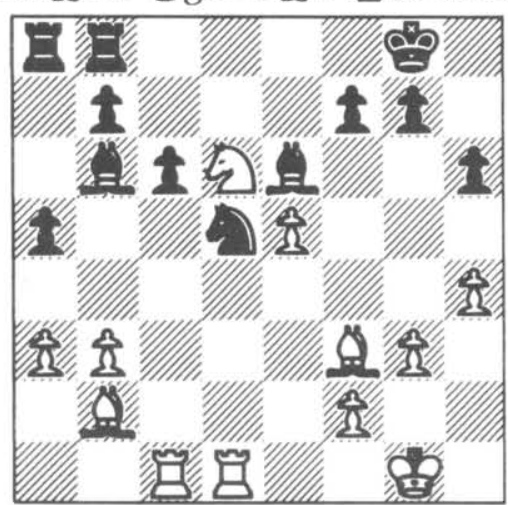

Position after $25 \ldots$ a5

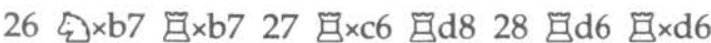

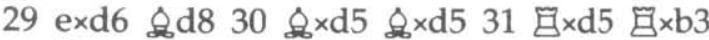

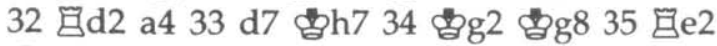

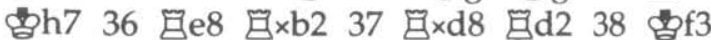

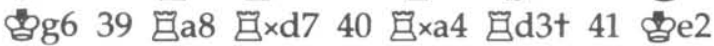

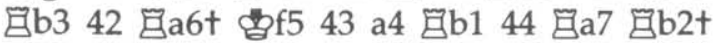

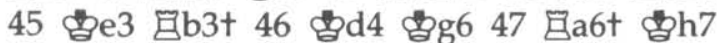

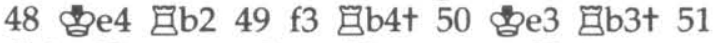

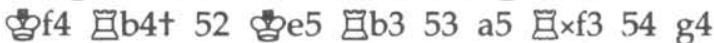

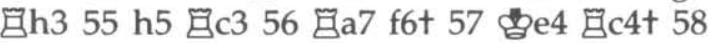

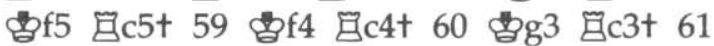

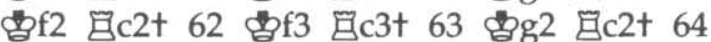

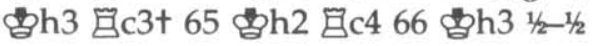

\section{Schach 2.7 - Cray Blitz D55/11 08 N:e4}

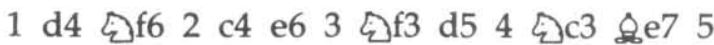

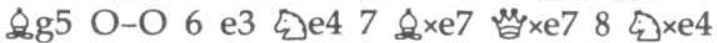

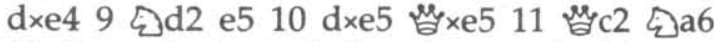
12 O-O-O \&g4 13 f3 exf3 14 g×f3 oh5 15 \&d3 f5 16 Ghe1 Gad8 17 \&f1 쌍.h2 $18 \mathrm{c5}$

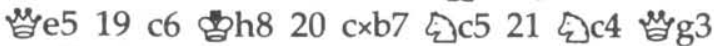

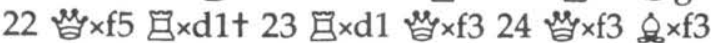

25 月d4 \&xb7 26 \&e2 g6 27 々e5 月e8 28

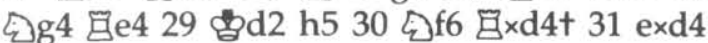

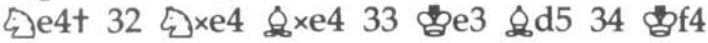
\&×a2 35 \&d3 bg7 36 bg5 \&f7 37 \&e4 a5 38 \&c6 \&g8 39 \&e4 \&h7 40 \&d3 h4 41 \&f1

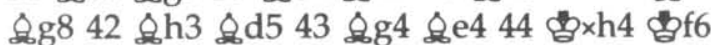
45 \&d7 \&f5 46 \&c6 g5t 47 gig3

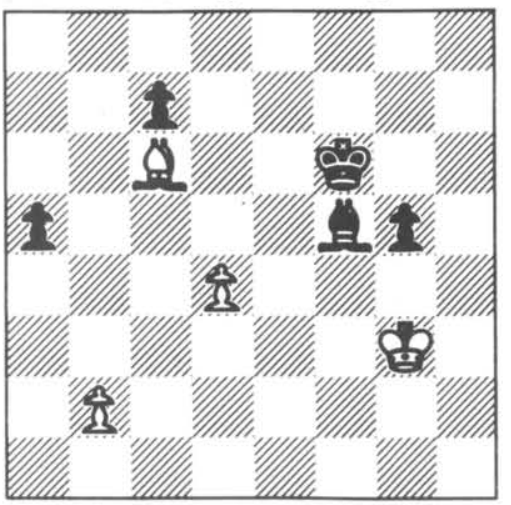

Position after 47 gg 3

0-1 (adjudicated)

Round 5

Cray Blitz - Hitech

D26/03 08. b5

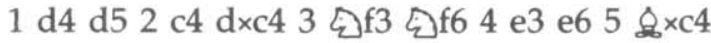
c5 6 랑e2 a6 7 d×c5 $8 \times c 58$ O-O b5 9 月d1

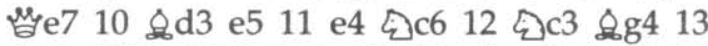

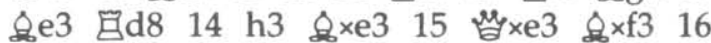

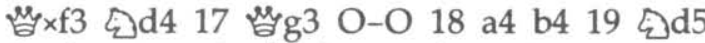

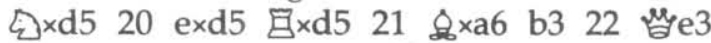

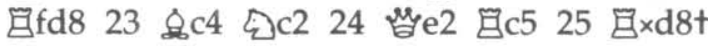
랑 $\times \mathrm{d} 8$

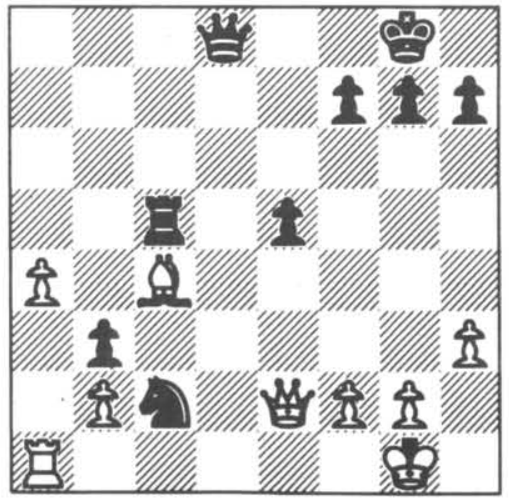

Position after $25 \ldots$... .

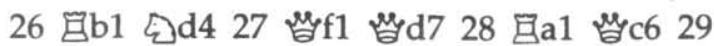

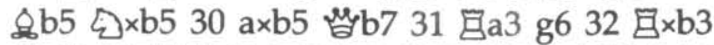

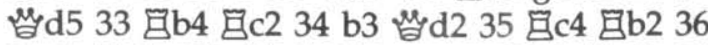

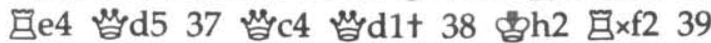

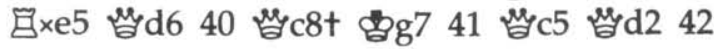




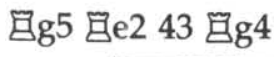

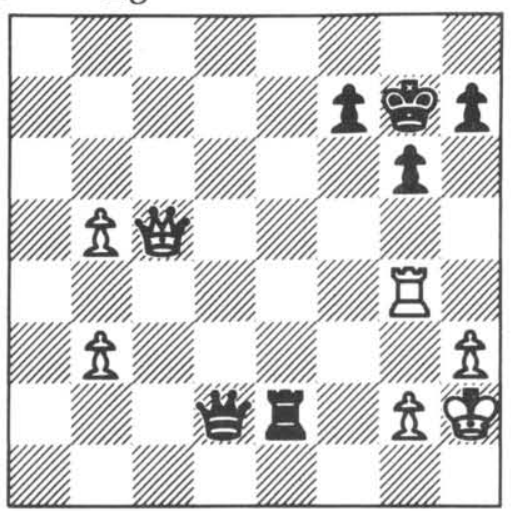

Position after 43 目4

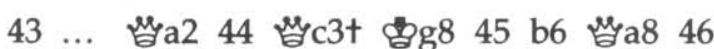

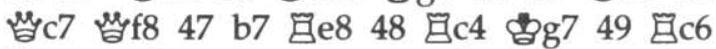

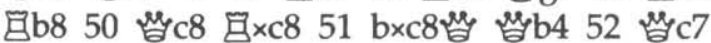

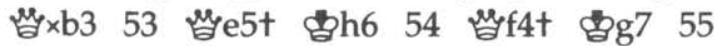

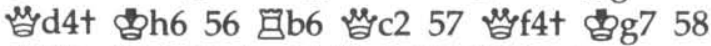

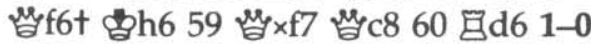

\section{Bobby - Sun Phoenix C00/02 06 O-O-O}

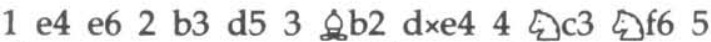
쓸 2 \&e7 6 O-O-O 쌈 447 ge1 O-O 8

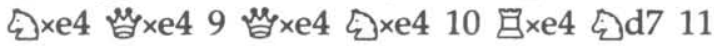

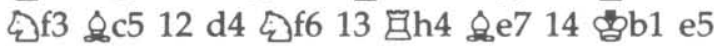
$15 \mathrm{~h} 3$ e4 16 ¿e5 \&e6 17 \&c4 \&×c4 18 bxc4 c5 19 d5 e3 20 日f4 exf2 21 日xf2 §e4 22 日f4

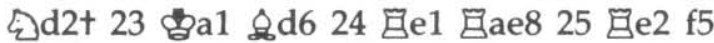

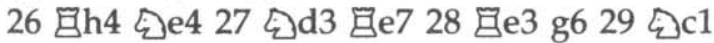

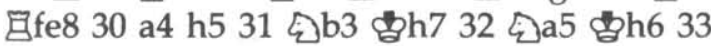

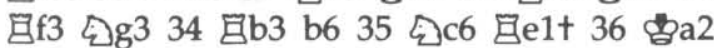

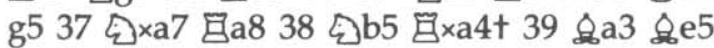
40 ¿d4 0-1

\section{Plymate - Lachex $\mathrm{C} 80 / 1409 \mathrm{Be} 3$}

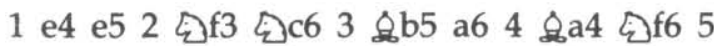
$\mathrm{O}-\mathrm{O}$ ¿ेe4 6 d4 b5 7 \&b3 d5 8 dxe5 $\$$ e6 9 \&e3 @ic5 10 쌈d2

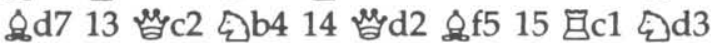

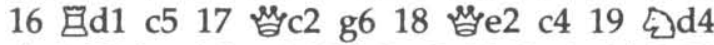
\& c8 20 b×c4 bxc4 21 b3 a5 22 ¿c3 \&b7 23

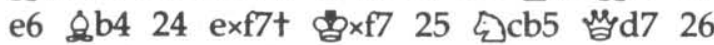

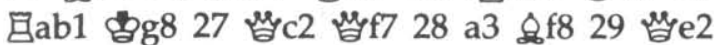

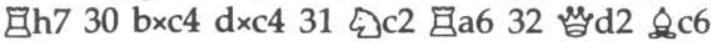

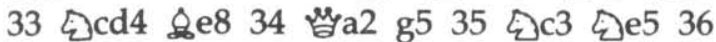

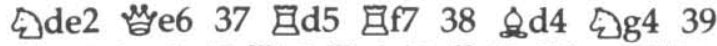

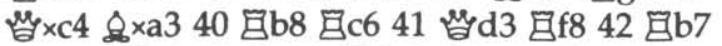

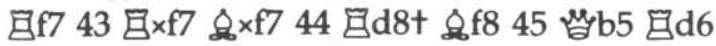

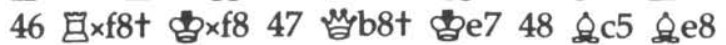

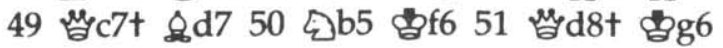

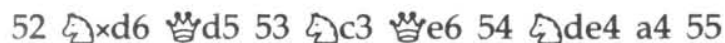

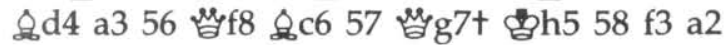
59 ¿ $3+$ thin 4

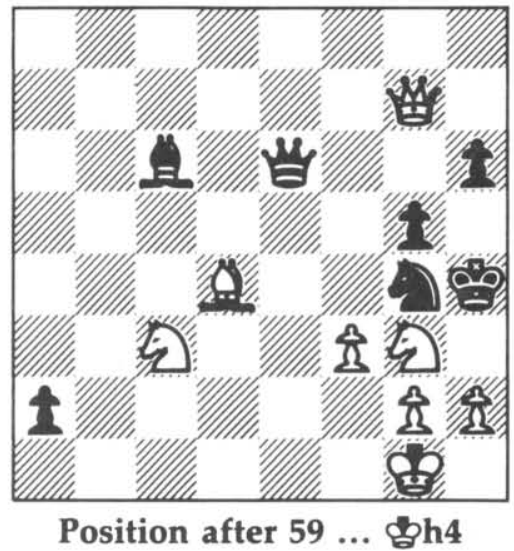

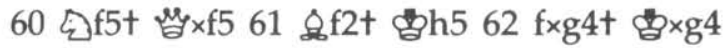
$63 \mathrm{~h} 3+\mathrm{gh} 564 \mathrm{~g} 4+1-0$

Rebel - Bebe
A65/09 07. a6

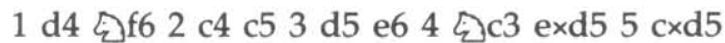

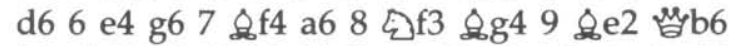
10 씸 2 \&g7 11 O-O O-O 12 h3 $2 \times f 313$ \&.f3 ¿bd7 14 Gad1 目fe8 15 b3 ¿e5 16

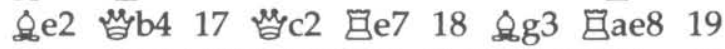
国fe1 g5 20 自f1

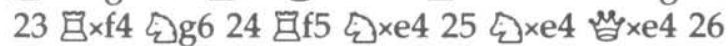

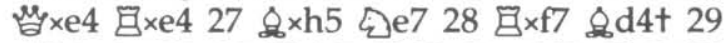

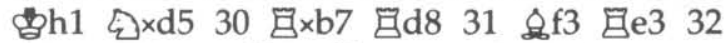

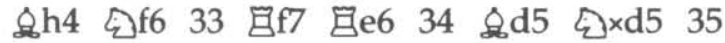

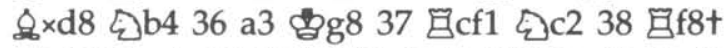
bg7 39 a4 d5 40 h4 乞e3 41 自1f7t tg6 42

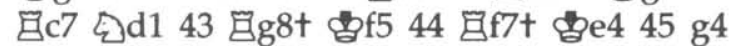

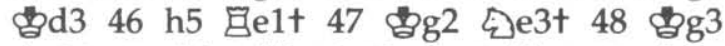

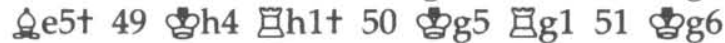

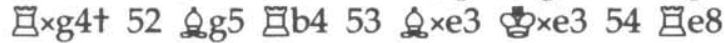

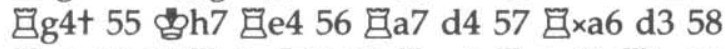

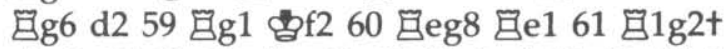

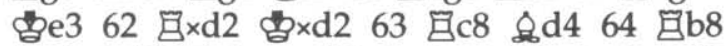

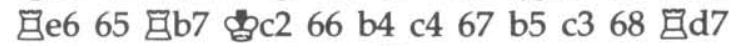
b्gid3 69 b6 c2 70 b7 c1쌈 0-1

$$
\begin{gathered}
\text { Dutch - Ostrich } \\
\text { A07/05 03. Bg4 }
\end{gathered}
$$

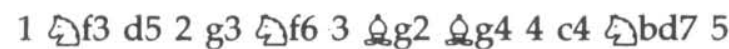

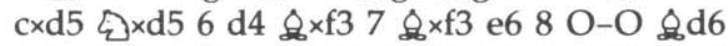

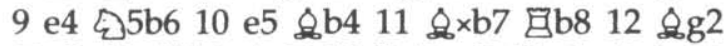

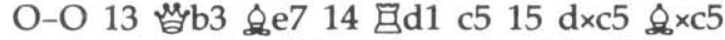

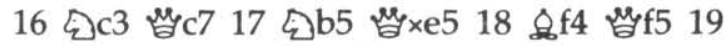

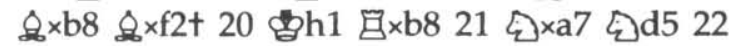

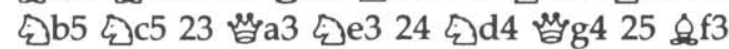

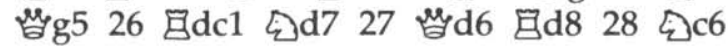


¿5 29 름3

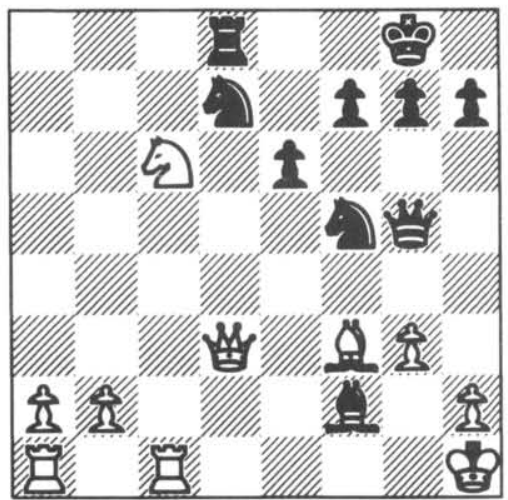

Position after 29 ․ㅏㅁ d3

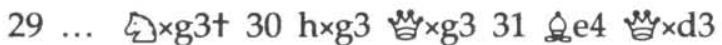

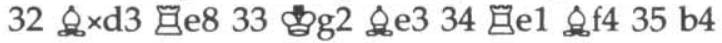
g5 36 a4 g4 37 目f1 e5 38 回ae1 冒6 39 b5

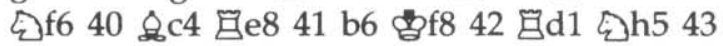
b7 e4 44 月d7 1-0

\section{Nona - Cyrus $68 \mathrm{~K}$ \\ C16/04 06. Be7}

1 d4 e6 2 e4 d5 3 乞c3 \&b4 4 e5 £e7 5 \&d2

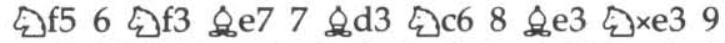

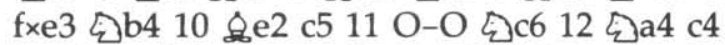

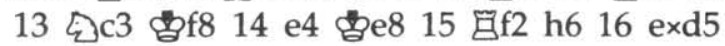

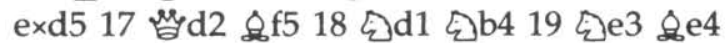
20 b3 cxb3 21 axb3 h5 22 ¿g5 \& $\times$ g5 23

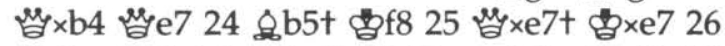
品1 be6 27 c4 目af8 28 cxd5t \&xd5 29

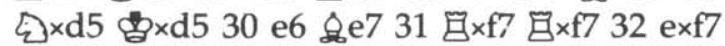
\&b4 33 月d1 自f8 34 \&e2 h4 35 \&c4t by

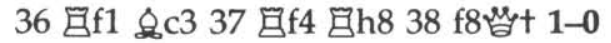

$$
\begin{gathered}
\text { Vaxchess - Chat } \\
\text { E73/03 06. Nc6 }
\end{gathered}
$$

1 d4 乞f6 2 c4 g6 3 乞c3 \&g7 4 e4 d6 5 \&e2

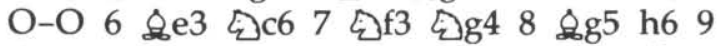

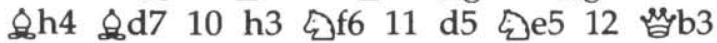
乞 $\times f 3+13 \& \times f 3$ b6 14 冒d1 e5 15 dxe6 \&c6 16

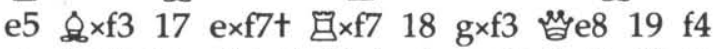

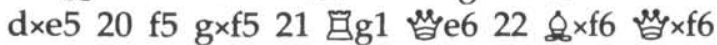

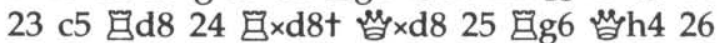

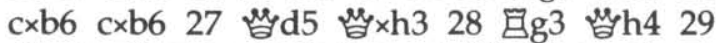

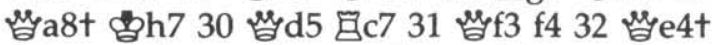

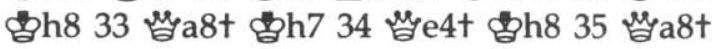
bh 36 쌈 $4+1 / 2-1 / 2$

\section{Mephisto - Schach 2.7 D30/01 03 g3}

1 d4 d5 2 c4 e6 3 g3 d×c4 4 kga4t \&d7 5

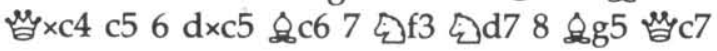

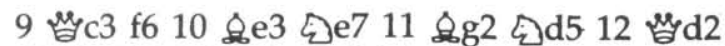

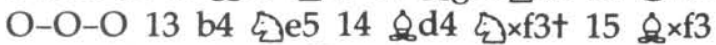

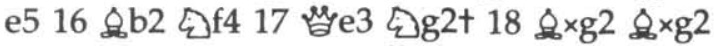

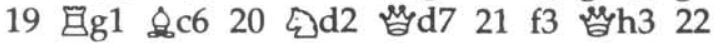

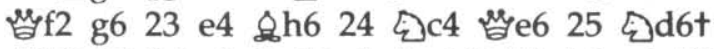
Bab8 26 b5 \&e8 27 \&a3 \&f7 28 b6 a6 29

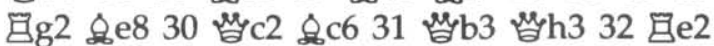

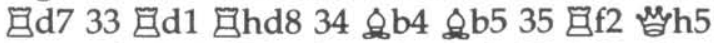
36 a4 \&c6 37 目d3 \&g7 38 \&c3 \&h6 39 씽 6 \&g7 40 a5 \&b5 41 回d1 \&a4 42 目a1 \&c6 43

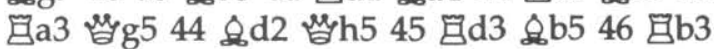
\&a4 47 月c3 \&c6 48 自c1 f5 49 目c2 fxe4 50 fxe4 \&a4 51 目c1 \&c6 52 h4 \&h6 53 \& 8 h6

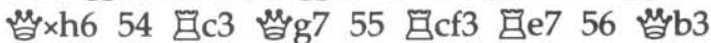

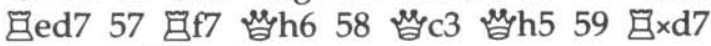
\&xd7 60 目f7 \&c6

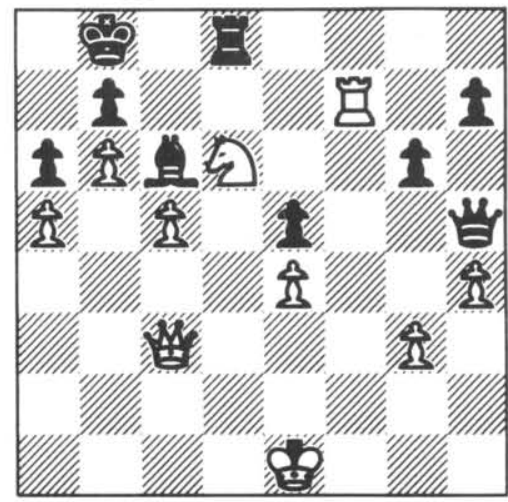

Position after $60 \ldots \& c 6$

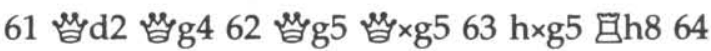
क्वd2 $8 \mathrm{~g} 8$ 1-0

\section{Advance 68K - Enterprise C80/04 15 Bf4}

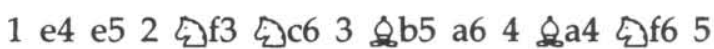

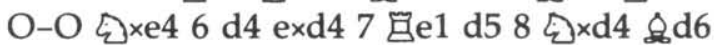

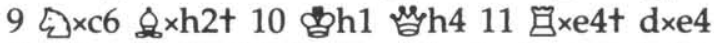

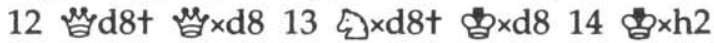
\&e6 15 \&f4 b5 16 \&b3 \&xb3 17 axb3 c6 18 ¿c3 f5 19 घe2 g6 20 घd4 हुd7 21 हd1

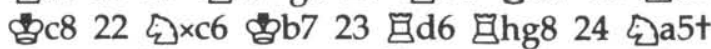

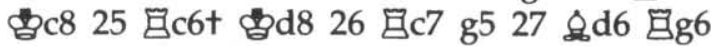

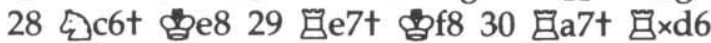

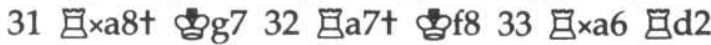

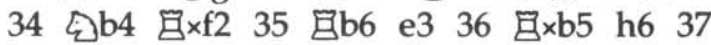

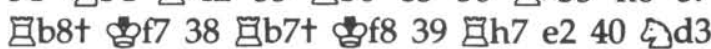

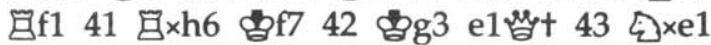

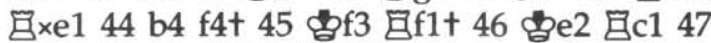

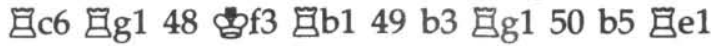

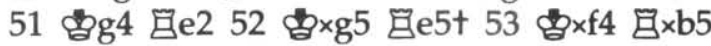

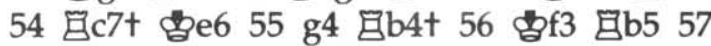
冒 6 十 冒 4t bd6 61 b4 回b7 62 c3 目7t 63 be3 


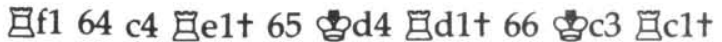

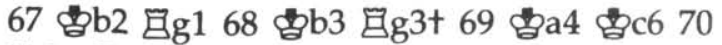

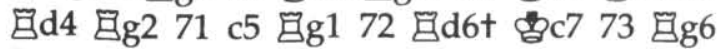

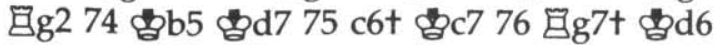
77 bुb6 1-0

$$
\begin{gathered}
\text { BCP - Awit } \\
\text { C45/01 04. Qf6 }
\end{gathered}
$$

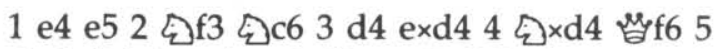

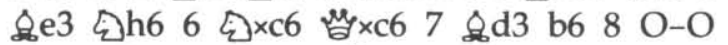

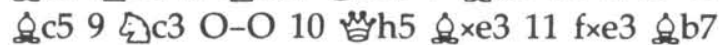

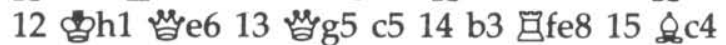

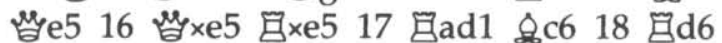
可8 19 h3 g6 20 \&d5 百c8 21 \& $8 \times c 6$ d×c6 22

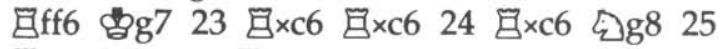

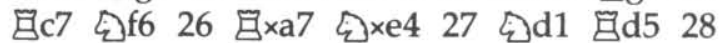
¿b2 $\begin{array}{ll} & \end{array}$

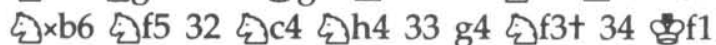

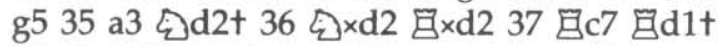

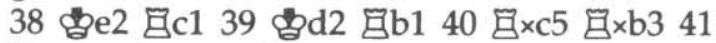

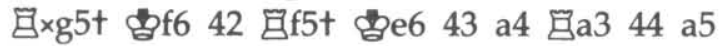

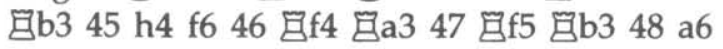

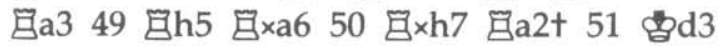

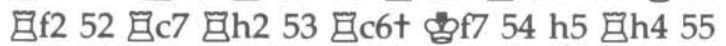

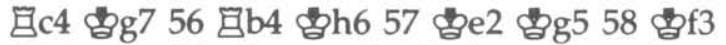

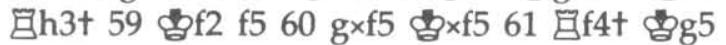

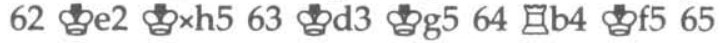

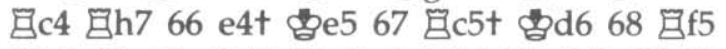

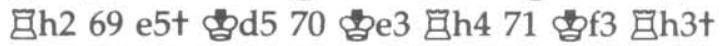

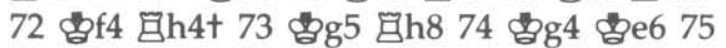

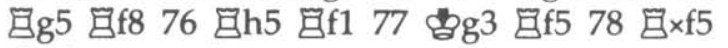

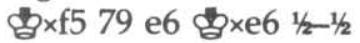

$$
\begin{gathered}
\text { Shess - Rex } \\
\text { C40/06 05. Qf7 }
\end{gathered}
$$

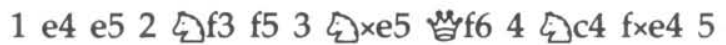

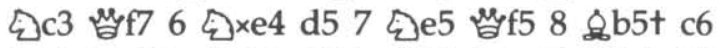

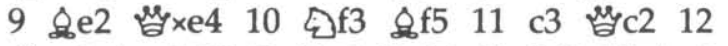

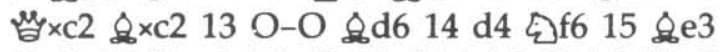
O-O 16 Gae1 $气 b d 717$ bुh1 b5 18 b3 国e8 19 c4 bxc4 20 b×c4 d×c4 21 \& $\times c 4+$ कुh 822 ¿g5 \&g6 23 f4 $2 \mathrm{~d} 5 \quad 24 \quad \& \times d 5$ cxd5 25 f5 \&b4 26 fxg6 \&×e1 27 \&f7t bुg8 $28 \mathrm{~g} \times \mathrm{h} 7 \mathrm{t}$

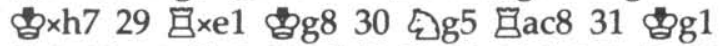

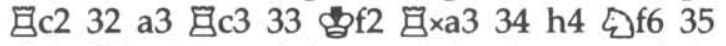
की

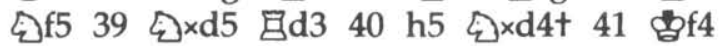

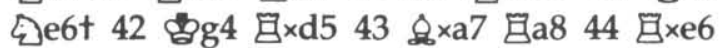

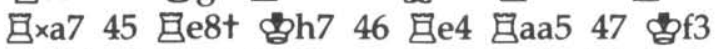

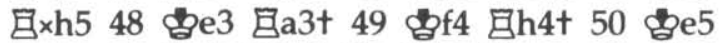

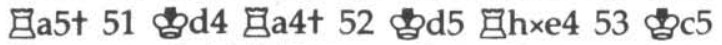

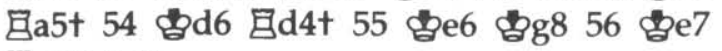
目5\# 0-1
Photo by L. Lindner

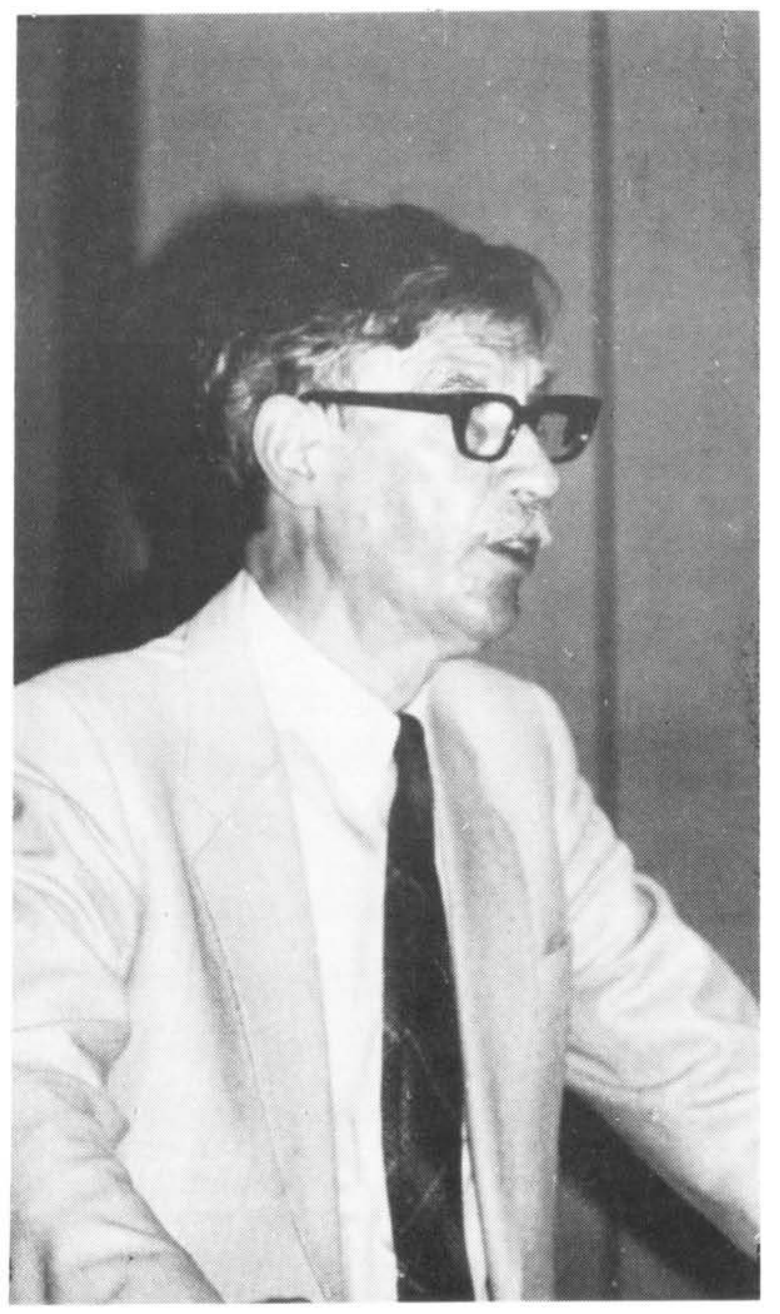

Still going strong on intuition.

Emeritus Professor Adriaan de Groot in Cologne. 